\title{
Long-term Transverse Imaging of the Hippocampus with Glass Microperiscopes
}

William T Redman ${ }^{1}$, Nora S Wolcott ${ }^{2}$, Luca Montelisciani ${ }^{3}$, Gabriel Luna ${ }^{4}$, Tyler D Marks ${ }^{4}$, Kevin K Sit ${ }^{5}$, Che-Hang Yü, Spencer L Smith ${ }^{4,6}$, and Michael J Goard ${ }^{2,4,5, *}$

${ }^{1}$ Interdepartmental Graduate Program in Dynamical Neuroscience, University of California, Santa Barbara,

Santa Barbara, CA 93106, USA

${ }^{2}$ Department of Molecular, Cellular, and Developmental Biology, University of California, Santa Barbara,

Santa Barbara, CA 93106, USA

${ }^{3}$ Cognitive and Systems Neuroscience Group,

University of Amsterdam,

Amsterdam, NL 1098 XH

${ }^{4}$ Neuroscience Research Institute,

University of California, Santa Barbara,

Santa Barbara, CA 93106, USA

5Department of Psychological and Brain Sciences,

University of California, Santa Barbara,

Santa Barbara, CA 93106, USA

${ }^{6}$ Department of Electrical and Computer Engineering,

University of California, Santa Barbara,

Santa Barbara, CA 93106, USA

*Correspondence to: michael.goard@lifesci.ucsb.edu 


\section{ABSTRACT}

2 The hippocampus consists of a stereotyped neuronal circuit repeated along the septal-temporal

3 axis. This transverse circuit contains distinct subfields with stereotyped connectivity that support

4 crucial cognitive processes, including episodic and spatial memory. However, comprehensive

5 measurements across the transverse hippocampal circuit in vivo are intractable with existing

6 techniques. Here, we developed an approach for two-photon imaging of the transverse

7 hippocampal plane in awake mice via implanted glass microperiscopes, allowing optical access

8 to the major hippocampal subfields and to the dendritic arbor of pyramidal neurons. Using this

9 approach, we tracked dendritic morphological dynamics on CA1 apical dendrites and

10 characterized spine turnover. We then used calcium imaging to quantify the prevalence of place

11 and speed cells across subfields. Finally, we measured the anatomical distribution of spatial

12 information, finding a non-uniform distribution of spatial selectivity along the DG-to-CA1 axis.

13 This approach extends the existing toolbox for structural and functional measurements of

14 hippocampal circuitry.

\section{INTRODUCTION}

17 The hippocampus is critical for episodic and spatial memories ${ }^{1-3}$, but the neural computations

18 underlying these functions are not well understood. The trisynaptic circuit linking entorhinal cortex (EC) to dentate gyrus (DG), DG to CA3, and CA3 to CA1 is believed to endow the hippocampus with its functional capabilities. Since the circuit was first described in the anatomical studies of Ramon y Cajal over a century $\mathrm{ago}^{4}$, considerable work has focused on each of the major hippocampal subfields (CA1-3 and DG) to identify their roles in hippocampal processing. The resulting body of literature has indicated that the subfields have related, but distinct roles in pattern separation and completion ${ }^{5-14}$, response to novelty ${ }^{15-19}$, and the encoding of social variables ${ }^{20-22}$. Additionally, there appear to be differences between the subfields in place field stability ${ }^{19,23,24}$. Although this work has increased our understanding of each of the subfields individually, it is not clear how neuronal activity is coordinated across the hippocampus.

This lack of knowledge comes, in part, from the technological limitations that prevent the 30 recording of neuronal populations across hippocampal subfields in the same animal.

31 Historically, electrophysiology has been the principal tool used to study the hippocampus.

32 Electrophysiological recordings have the advantage of high temporal resolution and can directly 33 measure spiking, but they are typically limited to small numbers of neurons in particular 34 subfields. Additionally, localization of recorded neurons within the hippocampus is approximate, 
requiring post-hoc histological analysis to estimate the position of the electrode tracks, with the distances between the recorded neurons and the electrode sites being poorly defined. In recent years, calcium imaging approaches (i.e. single photon mini-endoscopes and two-photon microscopy) have been used to record hippocampal activity, allowing for the simultaneous measurement of large numbers of neurons with known spatial relationships ${ }^{24-29}$. However, these approaches require aspiration of the overlying neocortex and are generally restricted to a single subfield for each animal.

Taken together, current experimental techniques are limited in their ability to: (1) record response dynamics and coordination across the hippocampus, (2) identify and distinguish between different neural subtypes, (3) allow for the chronic recording of cells across subfields, and (4) resolve key cellular structures, such as apical dendritic spines.

To address these challenges, we have developed a procedure for transverse imaging of the trisynaptic hippocampal circuit using chronically implanted glass microperiscopes. As has been found with previous studies using implanted microprisms in cortex ${ }^{30-33}$, the neural tissue remained intact and healthy for prolonged periods of time (up to 10 months), and both dendritic structure and calcium activity could be repeatedly measured in behaving mice. Optical modeling and point spread function measurements showed that axial resolution is decreased compared to traditional cranial windows, but is sufficient to image individual apical dendritic spines in hippocampal neurons several millimeters below the pial surface. Using this approach, we quantified spine turnover in CA1 apical dendrites across days, an experiment which has not been possible with existing methods. We then measured functional responses from CA1, CA3, and DG in head-fixed mice as they explored a floating carbon fiber arena ${ }^{34,35}$. We found neurons in all regions whose activity met criteria to be considered place cells (PC) and speed cells (SC). Further, we found a non-uniform distribution of spatial information across the extent of the DGto-CA1 axis. As we were able to simultaneously record from hundreds neurons along the DG-toCA1 axis with high spatial resolution, these results confirm findings from earlier electrophysiological studies that found similar heterogeneity ${ }^{36-38}$. Taken together, this approach adds to the existing neurophysiological toolkit and will enable chronic structural and functional measurements across the entire transverse hippocampal circuit.

\section{RESULTS}

66 Optical access to the transverse hippocampus using implanted microperiscopes

67 To image the transverse hippocampal circuit using two-photon (2P) imaging, we developed a

68 surgical procedure for chronically implanting a glass microperiscope into the septal 
(dorsomedial) end of the mouse hippocampus (Fig. 1A; Fig. S1; see Methods). For imaging CA1 only, we used a $1 \mathrm{~mm} \times 1 \mathrm{~mm} \times 2 \mathrm{~mm}$ microperiscope ( $\mathrm{v} 1_{\mathrm{CA} 1}$; Fig 1B, left), and for imaging the entire transverse hippocampus (CA1-CA3, DG), we used a $1.5 \mathrm{~mm} \times 1.5 \mathrm{~mm} \times 2.5 \mathrm{~mm}$ microperiscope (v2 HPC; Fig 1B, right). The microperiscope hypotenuse was coated with enhanced aluminum in order to reflect the imaging plane orthogonally onto the transverse plane (Fig. 1C, D). To insert the microperiscope, we made an incision through the dura and tissue (Fig. S1A), then lowered the tip of the microperiscope into the incision (Fig. S1B), pushing the cortical tissue medially (Fig. S1C). Although this approach eliminated the need for the aspiration of cortical tissue typically performed prior to hippocampal imaging ${ }^{25}$, it nonetheless results in severed connections and compressed tissue medial to the implant. Since the septal end of the hippocampus is affected by the implant, we used immunohistochemistry to quantify the effect of microperiscope implantation on microglia and astrocyte proliferation as a function of distance from the prism face (Fig. 1E). Similar to previous research using microprism implants ${ }^{31}$, we found an increase in the prevalence of astrocytes and microglia $<200 \mu \mathrm{m}$ from the microperiscope face, but the prevalence decreased past this distance and was indistinguishable from the control hemisphere 300-400 $\mu \mathrm{m}$ from the microperiscope face (Fig. 1F).

\section{Characterizing optical properties of microperiscope}

Use of the microperiscope requires imaging through several millimeters of glass, which could cause beam clipping or optical aberrations, potentially resulting in decreased signal intensity and optical resolution. However, the extent to which imaging through glass prisms affects optical signal quality is not well understood. To determine how our approach affects two-photon image properties, we modeled the expected optical properties and compared it to the experimentallydetermined signal intensity and point spread function measurements using fluorescent microspheres (Fig. 2A). To characterize signal intensity, we measured the minimum laser power necessary for saturation at the center of the microsphere, holding other imaging parameters constant (Fig. 2B). We found that signal intensity imaged through the microperiscope was reduced compared to a standard coverslip ( $\mathrm{v} 1_{\mathrm{CA} 1}$ microperiscope: $59.0 \% \pm 6.6 \%$; $\mathrm{v} 2 \mathrm{HPC}$ microperiscope: $67.2 \% \pm 8.5 \%$; mean \pm s.d.; Fig. $2 \mathrm{C}$ ). Compared to a standard cranial window using a $0.15 \mathrm{~mm}$ coverslip, we found that the lateral resolution of the microperiscope, measured as the full width at half maximum (FWHM) of the fluorescent microsphere profile, was similar (coverslip: $0.7 \mu \mathrm{m}$; v1 $1_{\mathrm{CA} 1}$ microperiscope: $1.0 \mu \mathrm{m}$; v2HPC microperiscope: $0.7 \mu \mathrm{m}$; Fig. 2D-G), while the axial resolution of the microperiscope was worse (coverslip: $3.0 \mu \mathrm{m}$; $\mathrm{v}_{\mathrm{CA} 1}$ 
that the decrease in axial resolution is predominantly due to clipping of the excitation beam, resulting in a reduction of the functional numerical aperture of the imaging system (theoretical point spread function of $1_{\mathrm{CA} 1}$ microperiscope: $10.9 \mu \mathrm{m}$; v2 $2_{\mathrm{HPC}}$ microperiscope: $7.7 \mu \mathrm{m}$; see Methods) rather than optical aberrations. As a result, use of adaptive optics did not significantly improve the axial resolution, though adaptive optics did improve the signal intensity by $40-80 \%$ (data not shown). Finally, we measured whether the optical properties changed across the microperiscope imaging plane, measuring the lateral and axial point spread function across the horizontal extent of the microperiscope (Fig. $2 \mathrm{H}$ ). We found that the resolution was mostly uniform, though the edges showed marginally poorer lateral resolution, and marginally improved axial resolution compared to the center of the window (Fig. 2l). Taken together, these results indicate that imaging through the microperiscope lowers signal intensity and axial resolution. Despite these effects, the quality is still sufficient to image individual HPC neurons (Fig. 1C) and sub-micron morphological structures (Fig. 3) with laser power well below the photodamage threshold.

\section{Resolving spines on the apical dendrites of CA1}

Dendritic spines are highly dynamic and motile structures that serve as the postsynaptic sites of excitatory synapses in the hippocampus ${ }^{39,40}$. Previous in vitro studies suggest a role for dendritic spines in structural and functional plasticity, but the transient and dynamic nature of these structures make them ideally suited to being studied in vivo ${ }^{41-43}$. Although existing techniques allow imaging of spines on the basal CA1 dendrites near the surface of the hippocampus ${ }^{43,44}$, imaging the apical dendritic spines of hippocampal neurons has not previously been possible. Using the microperiscope, we were able to track dendritic spine dynamics throughout the somato-dendritic axis of both CA1 and CA3 neurons in intact mice.

In order to visualize apical dendrites in CA1 neurons, we implanted a cohort of Thy1GFP-M mice, sparsely expressing GFP in a subset of pyramidal neurons ${ }^{45}$, with v1 $1_{\mathrm{CA} 1}$ microperiscopes (Fig. 3A; $n=7$ mice). We focused on CA1 apical dendrites, but both apical and basal dendrites could be imaged in CA1-3 neurons. Although it is theoretically possible to image DG dendritic structures using the microperiscope, the Thy1-GFP-M mouse line has dense expression throughout DG (Fig. 1C), which prevented clear identification of distinct processes. To resolve individual spines along the dendrite, high-resolution images were taken from several axial planes spanning the segment, and a composite image was generated using a weighted average of individual planes (Fig. 3B, C; see Methods). We reduced noise by filtering and 
136 binarizing and isolated dendrites of interest for tracking across days (Fig. 3B-D; Fig. S2A; see

137 Methods).

138 Previous studies have shown that dendritic spines fall into four major morphological

139 subtypes: filopodium, thin, mushroom, and stubby ${ }^{46-48}$. We found that our resolution was

140 sufficient to classify dendritic spines into their relative subtypes and evaluate density and

141 turnover based on these parameters (Fig. 3E, F; Fig. S2B). Consistent with previous studies, we

142 found a non-uniform distribution of dendritic spines: $30.4 \%$ thin, $41.0 \%$ stubby, $26.2 \%$

143 mushroom, and $2.3 \%$ filopodium $(F(3,100)=51.47, p<0.0001$, one-way ANOVA; Fig. 3E). The

144 low proportion of filopodium found in this and previous $2 \mathrm{P}$ imaging and histological studies ${ }^{49,50}$

145 (2-3\%), as compared to electron microscopy studies ${ }^{51,52}(\sim 7 \%)$, may result from the narrow

146 width of these structures causing them to fall below the detection threshold. Consistent with

147 previous work ${ }^{53-55}$, we found that particular classes, such as filopodium, had a high turnover

148 rate, while other classes were more stable across sessions $(F(3,100)=7.17, p<0.001$, one-

149 way ANOVA; Fig. 3F).

We found that total turnover dynamics reflect $15.0 \% \pm 2.0 \%$ spine addition and $13.0 \% \pm$

$1511.9 \%$ spine subtraction across consecutive days (Fig. $3 \mathrm{H}, \mathrm{I}$ ). We computed the survival fraction,

152 a measure indicating the fraction of spines still present from day one ${ }^{42-44}$. Although daily spine

153 addition and subtraction was $15.0 \%$ in our original analysis (Fig. $3 \mathrm{H}, \mathrm{I}$ ), the survival fraction

154 curve yields a more conservative estimate of turnover dynamics. Across 10 days, we found a

$15523.5 \%$ net loss in original spines (Fig. 3G), indicating that most spine turnover takes place within

156 an isolated population of transient spines. Both our cumulative turnover and survival fraction

157 results were similar to previous findings from basal dendrites in CA $1^{43,44}$, indicating that apical

158 and basal dendrites exhibit similar spine dynamics. Although we only tracked spine turnover for

159 up to 10 days, we found that we could identify the same dendritic processes over long time

160 periods (up to 150 days; Fig. S3), allowing for long-term longitudinal experiments tracking

161 isolated dendritic structures.

Recording place and speed cells in CA1, CA3, and DG

164 Much of the experimental work testing the hypothesized roles of CA1, CA3, and DG neurons

165 has come from place cell (PC) recordings ${ }^{7-9,13,56,57}$. While the results of these studies have been

166 instrumental, it has not been possible to measure activity throughout the transverse

167 hippocampal circuit in the same animal. We therefore investigated the ability of our

168 microperiscope to record from PCs in each of the hippocampal subfields during exploration of a

169 spatial environment. 
To measure functional responses, we implanted v2 HPC microperiscopes in transgenic mice expressing GCaMP6s in glutamatergic neurons ${ }^{58,59}$ (see Methods). As with the Thy1-GFP$\mathrm{M}$ mice, we were able to image neurons from CA1-CA3, and DG in the same animal (Fig. 4A, B; Video S1). In some cases, depending on microperiscope placement, we were able to record from all three areas simultaneously (Fig. S4). In all HPC subfields, we found normal calcium dynamics with clear transients (Fig. 4C, D; Videos S2, S3). The imaging fields remained stable and the same field could be imaged over 100 days later (Fig. 4E). In addition, microperiscope implantation allowed measurement of neural responses from mossy cells in the dentate gyrus (Figs. S4, S5A) and, depending on prism placement, simultaneous imaging of deep-layer cortical neurons in parietal cortex (Fig. S5B).

As $2 \mathrm{P}$ microscopy generally requires the animal to be head-fixed, the behavioral assays used to probe PC activity are limited. Previous work has made use of virtual reality (VR $)^{24,25}$, however it remains unclear how similar rodent hippocampal activity in real world environments is to that in $\mathrm{VR}^{60,61}$. To study PC activity in a physical environment, our head-fixed mice explored a carbon fiber arena that was lifted via an air table ${ }^{34}$ (Fig. 5A; see Methods). The mice were thus able to navigate the physical chamber by controlling their movement relative to the floor. Although this approach lacks the vestibular information present in real world navigation, it captures somatosensory and proprioceptive information missing from virtual environments. Moreover, recent work has found that place field width and single cell spatial information using this approach is comparable to the responses of free foraging animals ${ }^{35}$. For measurement of place fields, we allowed mice to navigate a curvilinear track over the course of 20-40 minutes (Video S4). As found previously ${ }^{35}$, using a curvilinear track allowed for robust sampling of the spatial environment and improved place field localization, though they could also be measured in the open field. In order to measure spatial properties of the hippocampal neurons independent of reward, we relied on exploration rather than active reward administration for sampling of the environment.

To characterize place fields, we recorded from neurons in CA1, CA3, and DG (CA1: $n=$ 1026; CA3: $n=832$; and DG: $n=463$ ) in transgenic mice with panexcitatory expression of GCaMP6s (Fig. 4B; $n=8$ mice). We found PCs in all three subfields, with a distribution that was in general agreement with previous $2 \mathrm{P}$ imaging experiments ${ }^{19,24}$ (Fig. 5C, F; CA1: 31.7\%; CA3: 24.5\%; DG: 17.7\%; see Methods) and with fields that spanned the entirety of the track (Fig. 5E).

201 The spatial information and place field width of the CA1 place cells were similar to those found 202 in a previous study using the same floating chamber design ${ }^{35}$. We found that spatial information 203 was highest in CA1 (Fig. 5H; CA1: $0.86 \pm 0.03$ bits/inferred spike; CA3: $0.74 \pm 0.04$ 
204 bits/inferred spike; DG: $0.62 \pm 0.05$ bits/inferred spike; mean \pm s.e.m.) and that place field

205 widths were comparable across the three regions (Fig. 5G; CA1: $18.3 \pm 0.4 \mathrm{~cm}$; CA3: $18.1 \pm$

$2060.5 \mathrm{~cm}$; DG: $18.7 \pm 0.7 \mathrm{~cm}$; mean \pm s.e.m.). As cells responsive to speed have recently been

207 found in the medial $E C^{62,63}$ and $C A 1^{63,64}$, we also identified neurons as speed cells (SCs) if their

208 activity was significantly related to running speed ${ }^{62}$ (see Methods). We found SCs in CA1, CA3,

209 and DG, with all areas having cells that showed both increased and decreased activity with

210 higher running speeds (Fig. 5D, F). Speed cells were most abundant in DG (Fig. 5F; CA1: 9.1\%;

211 CA3: 13.5\%; DG: 30.2\%), consistent with recent work that found it was possible to decode the

212 speed of freely moving animals from the activity of DG, but not $C A 1^{57}$.

\section{Distribution of PC properties along the DG-to-CA1 axis}

215 Recent work has suggested that PC properties are heterogeneously distributed along the extent of the DG-to-CA1 axis. In particular, place field width and spatial information have been found to vary among different subregions of CA3, with dorsal CA3 (dCA3)/CA2 having lower spatial information and larger place fields than medial CA3 (mCA3) ${ }^{36-38}$, and proximal CA3 having values that were most similar to $\mathrm{DG}^{13}$. Such distributions could be supported by known anatomical gradients in connectivity of $\mathrm{CA} 3^{65-69}$. However, given that these studies required separate animals for the recording of each location along the DG-to-CA1 axis, and that electrophysiology has limited spatial resolution along the transverse axis, we used the microperiscopes to measure these properties throughout the DG-to-CA1 axis in the same mice.

Using the v2 $2_{\text {HPC }}$ microperiscope, we simultaneously imaged from several hundred cells (range: 230-292 neurons; total: 1054 neurons) extending from pCA3 to pCA1 (Fig. 6A). Recordings from distinct imaging planes in different mice $(n=4)$ were compared by calculating the distance of individual cells from the inflection point of the DG-to-CA1 transverse axis (Fig; 6A; see Methods). In agreement with previous studies ${ }^{36-38}$, we found a non-uniform distribution of spatial information along this axis (Fig. 6B, C; $F(6,1030)=4.35, p=2.4 \times 10^{-4}$ General Linear F-test against a flat distribution with the same mean). In particular, we found that pCA3 cells had spatial information that was closer in value to those in DG (Fig. 6C; Fig. 5H), and that mCA3 had spatial information that was greater than dCA3/CA2 (Fig. 6B, C). We found that place field widths were smallest in mCA3, and largest in $\mathrm{dCA} 3 / \mathrm{CA} 2$, although we failed to find a

234 statistically significant non-uniform distribution with respect to place field width across the extent 235 of the DG-to-CA1 axis (Fig. S6; $F(4,236)=1.28, p=0.28$, General Linear F-test against a flat 236 distribution with the same mean). 


\section{DISCUSSION}

239 The microperiscope hippocampal imaging procedure we developed allows researchers, for the

240 first time, to chronically image neuronal structure and functional activity throughout the

241 transverse hippocampal circuit in awake, behaving mice. This approach builds on microprism

242 procedures developed for imaging cortex ${ }^{30-32}$, allowing multiple hippocampal subfields in the

243 same animal to be accessed optically. Using the microperiscope, we were able to resolve

244 spines on the apical dendrites of CA1 pyramidal cells and track them across time. Additionally,

245 we were able to characterize place cells (PCs) and speed cells (SCs) in all three hippocampal

246 subfields, and investigate their anatomical distribution across subfields.

\section{Comparison to other methods}

249 Historically, electrophysiology has been the principal tool used to study the hippocampus.

250 Electrophysiological recordings have much higher temporal resolution than calcium imaging and can directly measure spikes, but have limited spatial resolution. Our approach allows large scale imaging of neurons across multiple hippocampal subfields, with known spatial and morphological relationships. In addition, our approach allows genetically-controlled labeling of particular cell types, imaging of cellular structures such as dendrites and spines, and unequivocal tracking of functional and structural properties of the same cells across time, none of which are possible with existing electrophysiological approaches. hippocampus. These include gradient index (GRIN) lenses ${ }^{70,71}$, and cannulas that can be combined with both one-photon (1P) head-mounted microendoscopes ${ }^{26-28}$ and $2 \mathrm{P}$ imaging 24,25,29. These methods have been limited to horizontal imaging planes, making it difficult to image CA3 and DG, and intractable to image all three subfields in the same animal. While all of these methods cause damage to the brain, and some require the aspiration of the overlying cortex, the damage is largely restricted to superficial hippocampus. This contrasts with the implantation of our microperiscope, which is inserted into the septal end of the hippocampus and necessarily causes some damage to the structure. Despite this, we find normal response properties, including selectivity for location and speed, in CA1-CA3 and DG (Fig. 5). Damage in the direction orthogonal to the transverse axis caused by the implantation of the microperiscope is similar to that caused by microprisms in cortex ${ }^{31}$, as glial markers were found to decay to

269 baseline levels 300-400 $\mu \mathrm{m}$ away from the face of the microperiscope. We showed that, 270 following successful implantation, the imaging fields were stable, and the same cells could be 271 imaged up to 3 months later (Fig. 4E). However, we emphasize that tissue damage caused by 
272 the microperiscope assembly to the hippocampus should be taken into consideration when

273 planning experiments and interpreting results.

274 We found that imaging through the microperiscope had some effect on signal intensity

275 and axial resolution. The effect depended not only on the path length through the glass, but also

276 on the geometry of the microperiscope. For example, the larger v2 $2_{\text {HPC }}$ microperiscope had

277 slightly better optical properties than the smaller v1 $1_{\mathrm{CA} 1}$ microperiscope (Fig. 2). The optical

278 properties should be considered along with the potential tissue damage during the planning of

279 experiments. Moreover, further customizing the microperiscope geometry for particular

280 applications may help optimize signal quality and minimize damage to neural structures.

Structural and functional properties along the transverse hippocampal circuit

We utilized the microperiscope in two experiments that would not have been possible with existing methods: (1) we tracked the spines on apical CA1 dendrites in vivo (Fig. 3); (2) we simultaneously recorded from PCs along the extent of the DG-to-CA1 transverse axis (Fig. 4).

Several studies have tracked the spines of basal CA1 dendrites in vivo by imaging the dorsal surface of the hippocampus ${ }^{43,44}$. However, spines on the apical dendrites have not been tracked in vivo. Given that these spines make up the majority of the input to CA1 pyramidal neurons, there is a significant need for understanding their dynamics. Using the microperiscope, we tracked isolated apical dendrites for up to 10 consecutive days (Fig. 3D). We found moderate addition and subtraction across days, indicating dynamic turnover in apical dendrites $($ Fig $3 \mathrm{H}, \mathrm{I})$. However, consistent with previous studies in basal spines ${ }^{43,44}$, we found the majority of spines (76.5\%) survived throughout the imaging period (Fig. 3G). This high survival fraction suggests that the cumulative turnover rates we observe are reflective of a distinct pool of transient spines, while the majority of spines remain stable across days over a longer timescale. These results add to the growing notion that, even in the absence of salient learning and reward signals, dendritic spines are dynamic structures ${ }^{43,72,73}$. Understanding the nature and timescales of these dynamics has significant implications for the reported instability of $\mathrm{PCs}^{23,24,27,74-76}$.

Previous studies have found gradients of connectivity in $C A 3^{65-69}$, suggesting that there may be functional gradients as well. Electrophysiological recordings have indeed found that spatial information and place field width vary as a function of distance along the DG-to-CA1 302 axis $^{36-38}$, and that the most proximal part of CA3 is functionally more similar to DG than to the 303 rest of $\mathrm{CA}^{13}$. This has led to a more nuanced understanding of the hippocampal circuit, with 304 coarse anatomical subdivisions having finer functional subdivisions. However, given that these 305 previous studies relied on recordings in different animals for each location along the DG-to-CA1 
axis, and given electrodes' poor spatial resolution in the transverse axis, the results have had to be cautiously interpreted. Using the microperiscope, we imaged several hundred neurons in multiple mice along the extent of the DG-to-CA1 axis (Fig. 6A). The location of each neuron relative to the CA3 inflection point could be easily identified, allowing for unequivocal characterization of spatial information and place field width along the DG-to-CA1 axis across several mice (Fig. 6B, C; Fig. S6). Similar to the previous studies ${ }^{36-38}$, we found a non-uniform distribution of spatial information (Fig. 6B, C). Place field width also appeared to vary along the DG-to-CA1 axis, though we failed to find statistically significant non-uniformity with respect to place field width (Fig. S6B). Indeed, we found that spatial information, but not place field width, differed significantly between CA1, CA3, and DG (Fig. 5H). Given the high spatial resolution and ability to simultaneously record from cells across the DG-to-CA1 axis using this approach, our results strengthen the hypothesis of distributed spatial coding across hippocampal subfields.

\section{Future applications}

This paper explored a few possible uses for the microperiscope in interrogating the hippocampal circuit. However, there are a number of candidate applications we did not pursue that could reveal novel insight into hippocampal function. Here, we describe several of these, chosen to highlight the utility of the microperiscope in addressing questions that are challenging or intractable with existing approaches: (1) The microperiscope allows for the recording from multiple hippocampal subfields simultaneously (Fig. S4), allowing investigation of interactions between neurons in different subfields during behavior. This could also be useful for determining the effect of neuron- or subfield-specific optogenetic manipulations on downstream subfields. (2) The microperiscope enables the investigation of local circuits by allowing morphological and genetic identification of different cell types. This includes identifying genetically-distinct hippocampal neurons (e.g. CA2 neurons, specific interneuron subtypes) as well was identifying particular cell types by position or morphological characteristics (e.g. mossy cells in DG; Fig. $\mathrm{S} 5 \mathrm{~A})$. As these distinct cell types play important roles in hippocampal function ${ }^{20,77-81}$, having access to them will enable a greater understanding of the hippocampal circuit. (3) The

334 microperiscope can be combined with retrograde-transported viruses to allow projection-based

335 cell identification, making it possible to identify neurons that project to specific downstream

336 targets. Since hippocampal neurons that project to different brain regions have been found to

337 exhibit distinct functional properties (e.g. neurons in ventral CA1 that project to the nucleus

338 accumbens shell have been implicated in social memory ${ }^{21}$ ), the union of these tools will be a

339 powerful means for understanding hippocampal outputs. (4) Finally, the microperiscope 
340 provides access to the entire dendritic tree of pyramidal neurons (Figs. 1C, 3A, 5B; Video S2),

341 giving optical access to dendritic signaling over a much larger spatial extent than has been

342 previously possible ${ }^{82-84}$. By sparsely expressing calcium or glutamate sensors in hippocampal

343 pyramidal neurons, spines throughout the somatodendritic axis could be imaged, allowing

344 determination of how place field responses arise from the responses of individual spines, similar

345 to experiments in visual cortex investigating the cellular origin of orientation tuning from synaptic

346 inputs $^{85,86}$.

347 Combined with electrophysiological and traditional imaging approaches, imaging of the

348 transverse hippocampal circuit with microperiscopes will be a powerful tool for investigating

349 hippocampal circuitry, structural dynamics, and function. 
bioRxiv preprint doi: https://doi.org/10.1101/2021.08.10.455885; this version posted November 8, 2021. The copyright holder for this preprint (which was not certified by peer review) is the author/funder, who has granted bioRxiv a license to display the preprint in perpetuity. It is made available under aCC-BY-NC 4.0 International license.

\section{FIGURES}

A

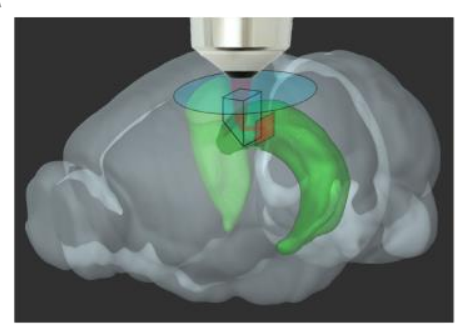

B

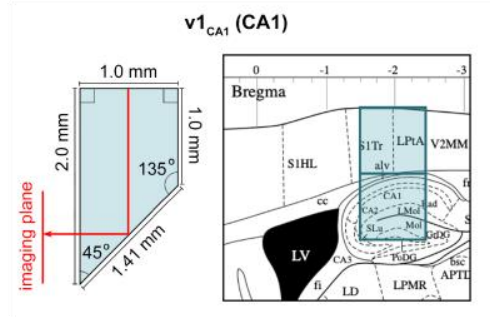

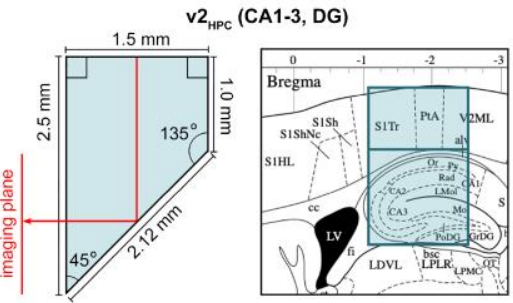

D

C

Thy1-GFP-M

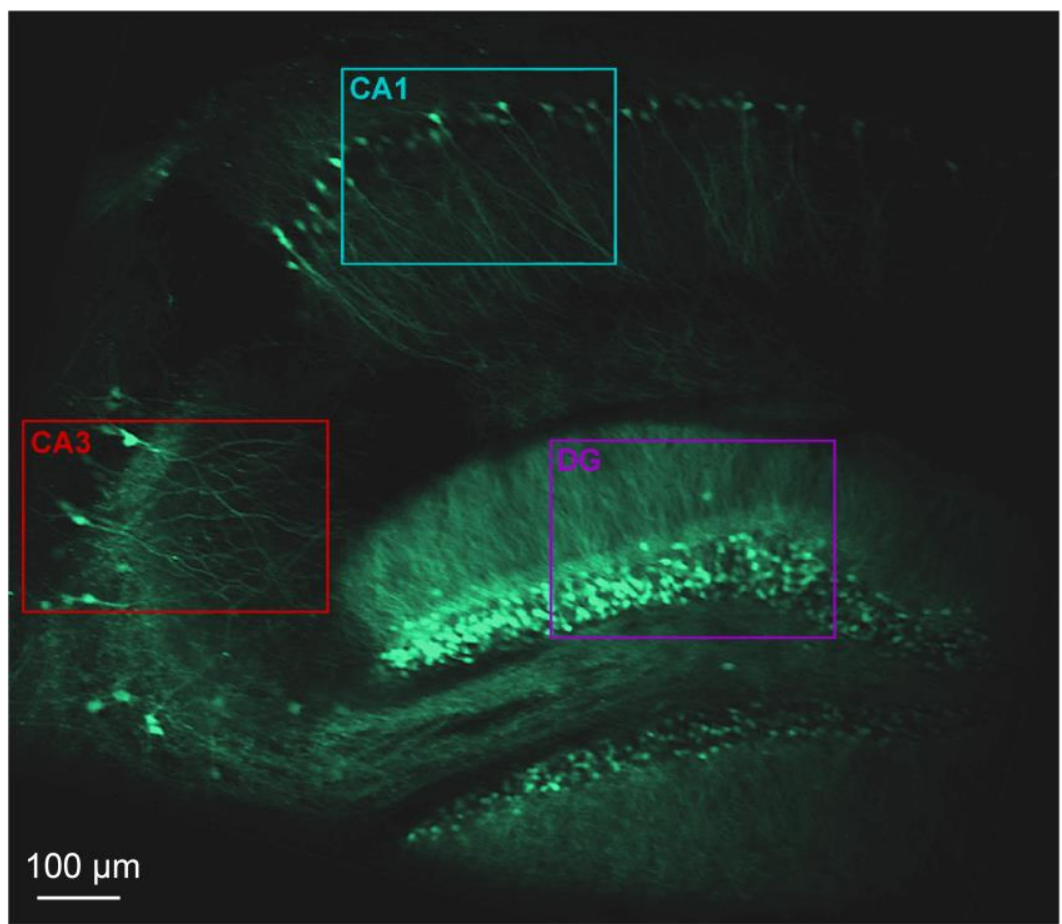

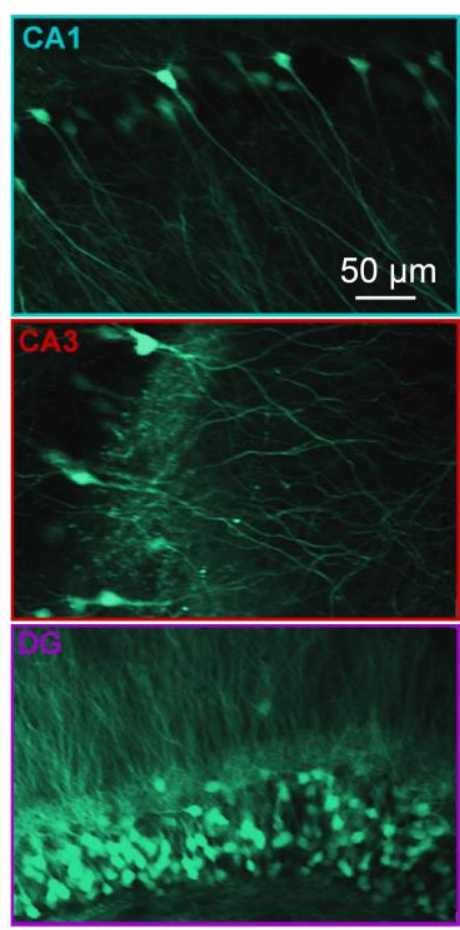

$\mathrm{F}$

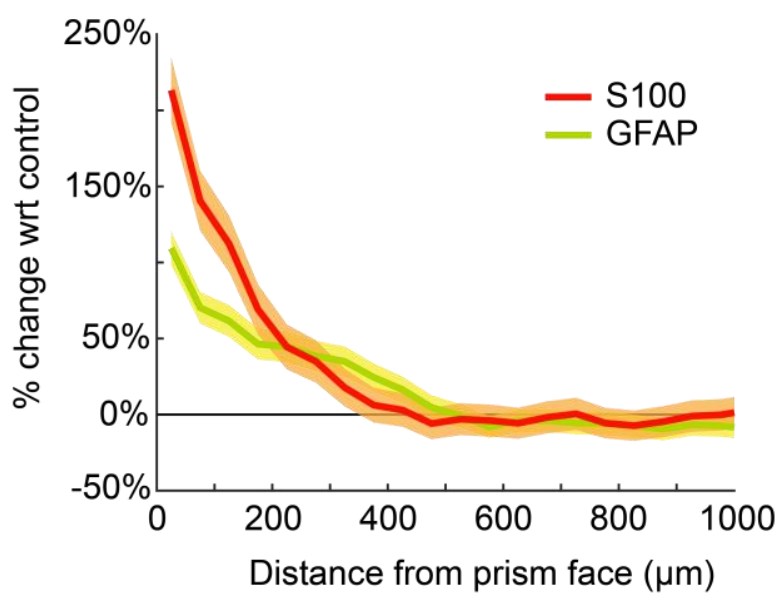


Figure 1. Implanted microperiscopes allow imaging of the hippocampal transverse plane.

(A) Three-dimensional schematic ${ }^{87}$ illustrating microperiscope implantation and light path for hippocampal imaging.

(B) Schematics ${ }^{88}$ showing the imaging plane location of $1_{\mathrm{CA} 1}(1 \mathrm{~mm}$ imaging plane, $2 \mathrm{~mm}$ total length) and $\mathrm{v} 2_{\mathrm{HPC}}(1.5 \mathrm{~mm}$ imaging plane, $2.5 \mathrm{~mm}$ total length) microperiscopes.

(C) Tiled average projection of the transverse imaging plane using the v2 $2_{\text {HPC }}$ microperiscope implant in a Thy1-GFP-M transgenic mouse. Scale bar $=100 \mu \mathrm{m}$.

(D) Enlarged images of hippocampal subfields (CA1, CA3, DG) corresponding to the rectangles in (C). Scale bar $=50 \mu \mathrm{m}$.

(E) Example histological section stained for microglia (S100 - red) and astrocytes (GFAP green). Scale bar $=300 \mu \mathrm{m}$.

(F) Quantification of microglia and astrocyte density as a function of distance from the prism face, normalized to the density in the unimplanted contralateral hemisphere $(n=2$ mice; mean \pm bootstrapped s.e.m.). 
bioRxiv preprint doi: https://doi.org/10.1101/2021.08.10.455885; this version posted November 8, 2021. The copyright holder for this preprint (which was not certified by peer review) is the author/funder, who has granted bioRxiv a license to display the preprint in perpetuity. It is made available under aCC-BY-NC 4.0 International license.

A

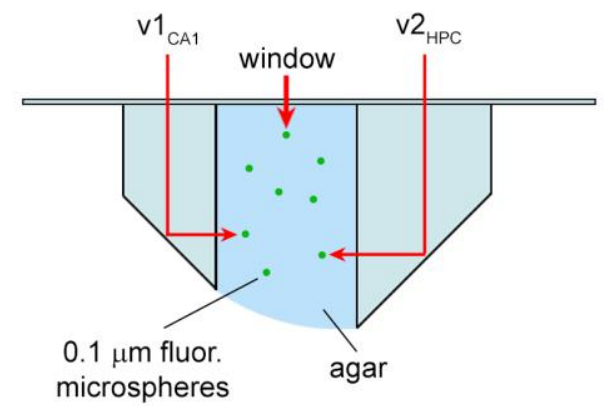

D

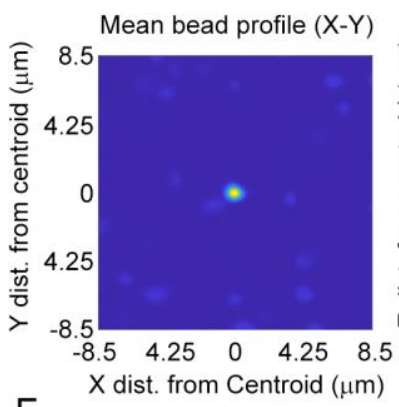

$\mathrm{F}$

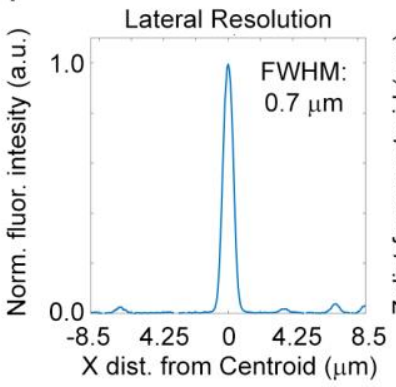

Coverslip
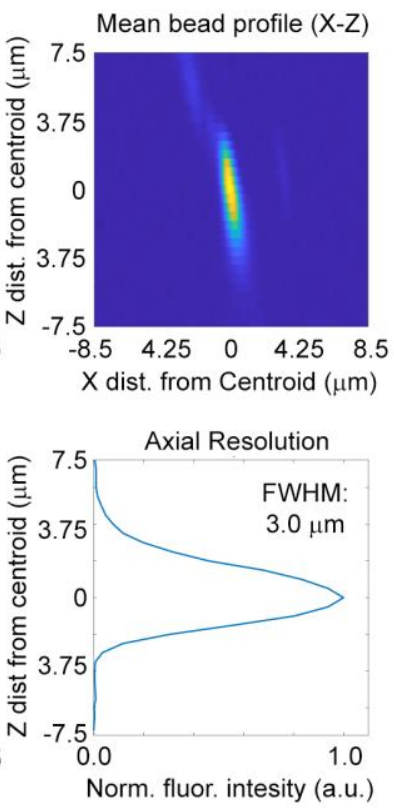
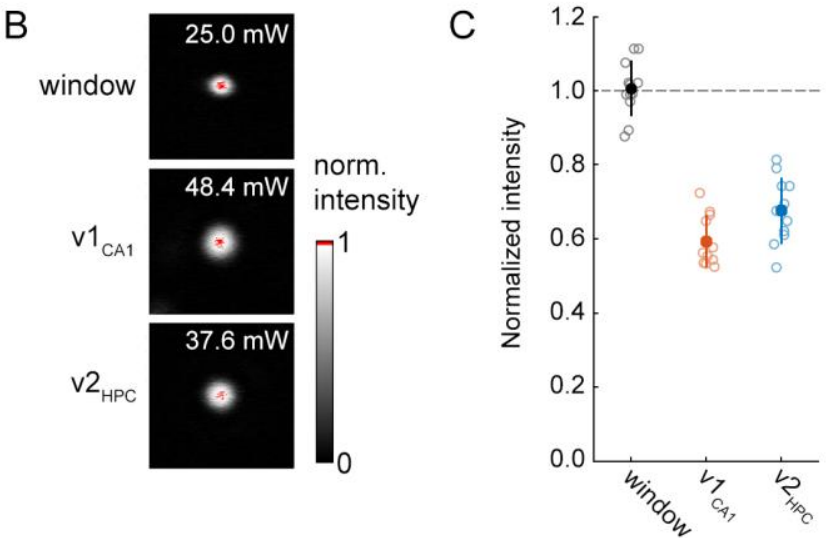

E

Microperiscope

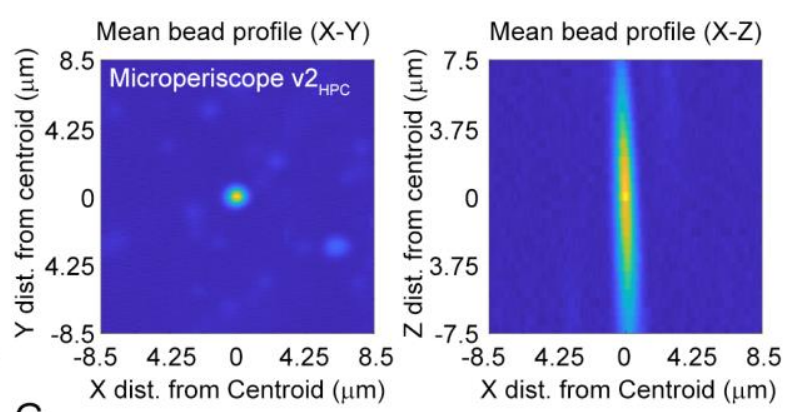

G
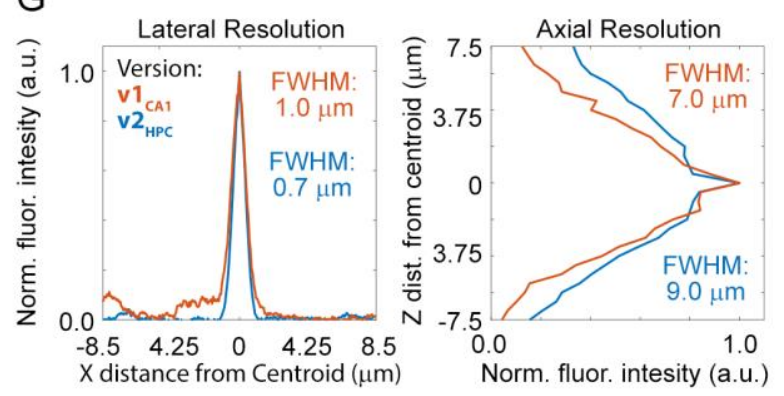

Lateral Resolution

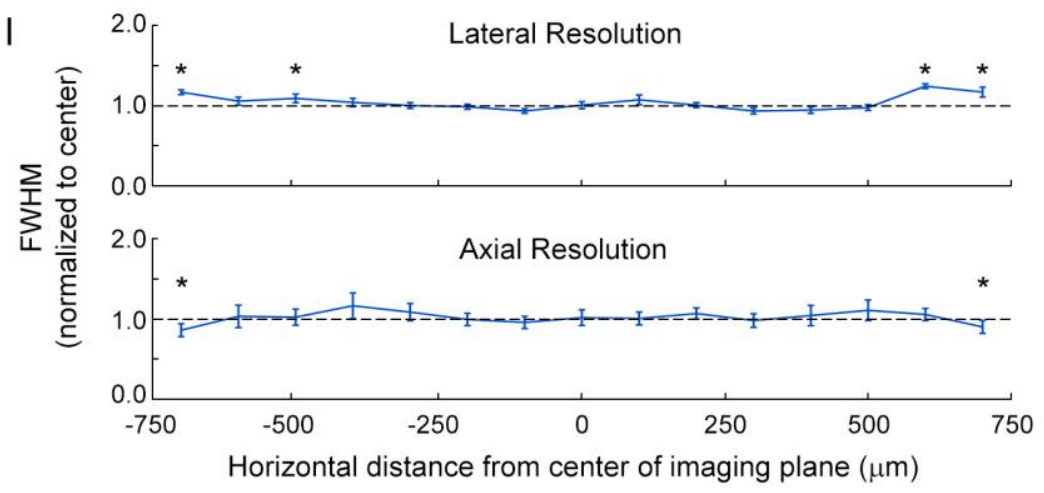


Figure 2: Optical characterization of cranial window and microperiscopes.

(A) Schematic of signal yield and resolution characterization experiments to allow comparison of microperiscopes ( $1_{\mathrm{CA}_{1}}$ and $\left.\mathrm{v} 2_{\mathrm{HPC}}\right)$ and standard coverslip window $(0.15 \mathrm{~mm}$ thickness) using $0.1 \mu \mathrm{m}$ fluorescent microspheres imaged with a 16x/0.8 NA objective.

(B) Example microspheres imaged through the window, v1 $1_{\mathrm{CA} 1}$ microperiscope, and v2 $\mathrm{HPC}$ microperiscope. Laser power was gradually increased to the minimum level necessary to saturate the centroid of the microsphere with all other imaging parameters held constant.

(C) Distribution of signal intensity values ( $n=12$ microspheres for each condition), measured as the reciprocal of the minimum laser intensity for saturation, and normalized to the mean intensity through the window ( $\mathrm{v} 1_{\mathrm{CA} 1}: 59.0 \% \pm 6.6 \%$; $2_{\mathrm{HPC}}: 67.2 \% \pm 8.5 \%$, mean \pm s.d.).

(D) Average X-Y profile (left) and X-Z profile (right) of fluorescent microspheres $(n=17$ microspheres) imaged through the window.

(E) Average X-Y profile (left) and X-Z profile (right) of fluorescent microspheres $(n=20$ microspheres) imaged through the $2_{\mathrm{HPC}}$ microprism $(2.5 \mathrm{~mm}$ path length through glass).

(F) Plot of normalized fluorescence intensity profile of $X$ dimension (lateral resolution; $F W H M=$ $0.7 \mu \mathrm{m}$ ) and $Z$ dimension (axial resolution; FWHM $=3.0 \mu \mathrm{m}$ ) through the centroid of the microsphere ( $n=17$ microspheres) imaged through the window.

(G) Plot of normalized fluorescence intensity profile of $X$ dimension (lateral resolution; v1 $1_{C A 1}$ $\mathrm{FWHM}=1.0 \mu \mathrm{m}$, orange; $2_{\mathrm{HPC}} \mathrm{FWHM}=0.7 \mu \mathrm{m}$, blue) and $\mathrm{Z}$ dimension (axial resolution; $\mathrm{V} 1_{\mathrm{CA} 1} \mathrm{FWHM}=7.0 \mu \mathrm{m}$; $\left.\mathrm{v} 2_{\mathrm{HPC}} \mathrm{FWHM}=9.0 \mu \mathrm{m}\right)$ through the centroid of the microsphere $(n=$ 10 microspheres for $\mathrm{v} 1_{\mathrm{CA} 1}, n=20$ microspheres for $\mathrm{v} 2_{\mathrm{HPC}}$ ).

$(\mathrm{H})$ Schematic of the experiment used to characterize lateral and axial resolution across the horizontal axis of the prism.

(I) Lateral (top) and axial (bottom) point spread function of average microsphere profile versus horizontal distance from the center of the imaging plane (mean $\pm 99 \%$ confidence interval of gaussian fit of mean microsphere profile), with point spread function values normalized to the mean at the center of the imaging plane $(-250$ to $+250 \mu \mathrm{m})$. Asterisks indicate that $99 \%$ confidence intervals are outside of the mean of the center points. 

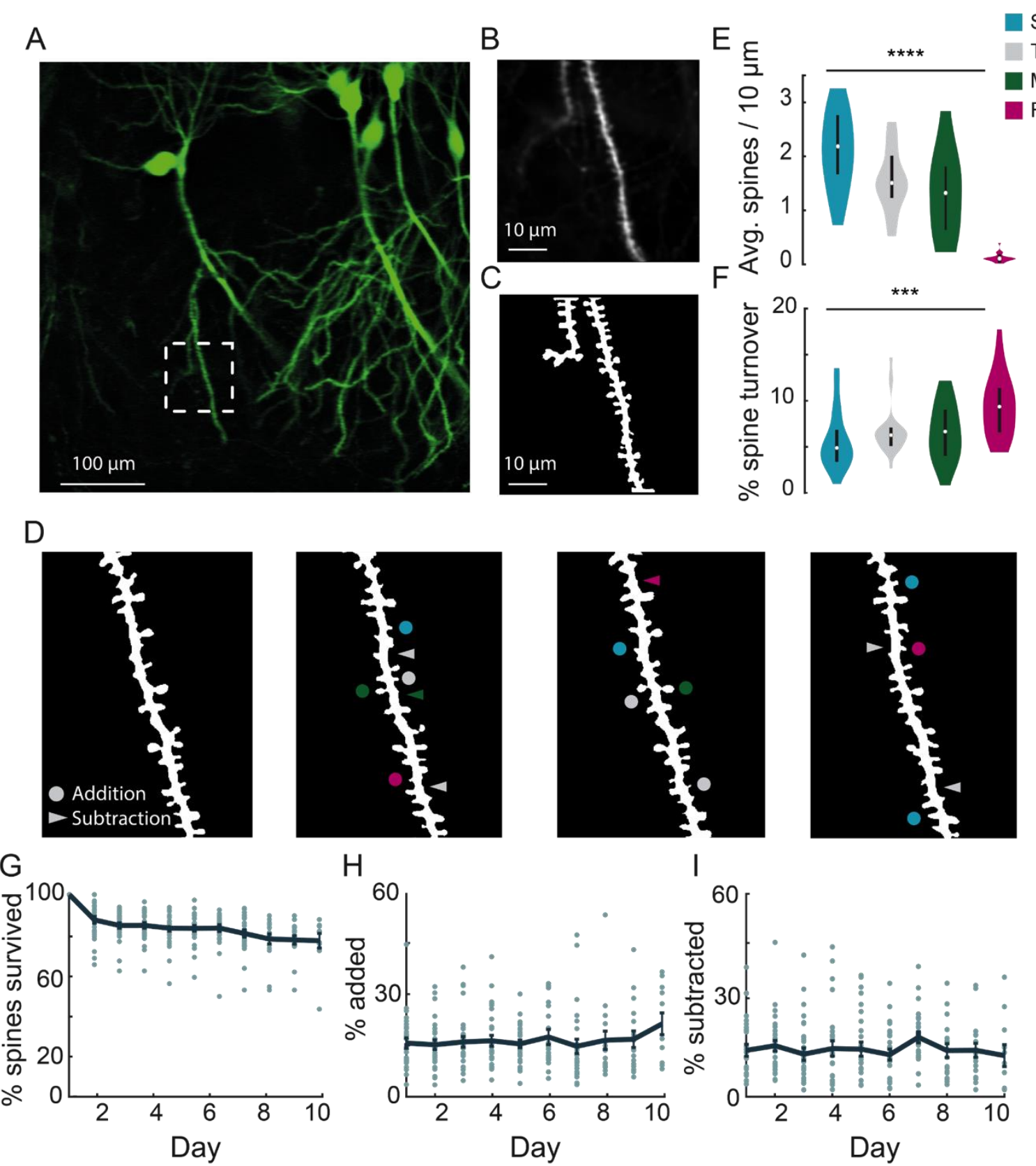

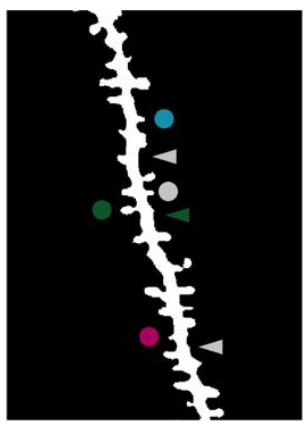

$\mathrm{H}$

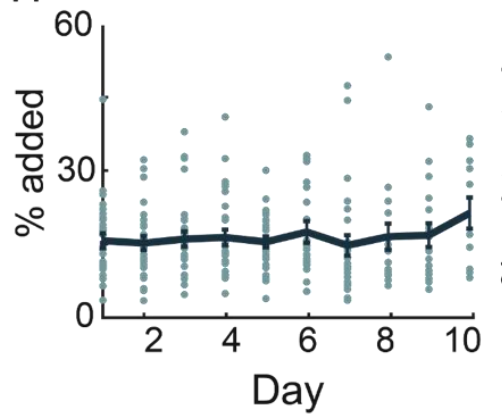

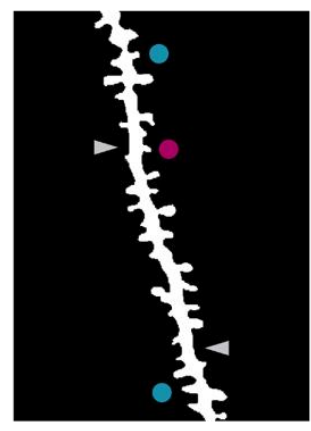

I

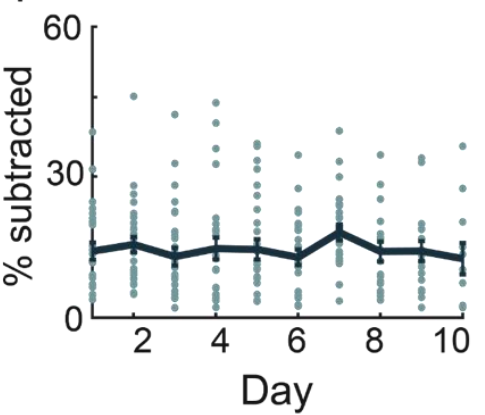

Figure 3. Chronic imaging of spine morphology in CA1 apical dendrites.

(A) Average projection of CA1 neurons sparsely expressing a GFP reporter (Thy1-GFP-M) imaged through the $1_{\mathrm{CA} 1}$ microperiscope. Scale bar $=100 \mu \mathrm{m}$.

(B) Weighted projection (see Methods) of the apical dendrites shown in the dashed box of (A). Scale bar $=10 \mu \mathrm{m}$.

(C) Filtered and binarized image (Figure S2A; see Methods) of the dendrites in (B) to allow identification and classification of individual dendritic spines. Scale bar $=10 \mu \mathrm{m}$. 
(D) Tracking CA1 dendritic spines over consecutive days on a single apical dendrite.

Arrowheads indicate subtracted spines and circles indicate added spines. Colors indicate spine type of added and subtracted spines: filopodium (magenta), thin (grey), stubby (blue), and mushroom (green).

(E) Average number of spines per $10 \mu \mathrm{m}$ section of dendrite, for each of the four classes of spine ( $n=26$ dendrites from 7 mice). one-way ANOVA, $F(3,100)=51.47,{ }^{* * *} p<0.0001$

(F) Percent spine turnover across days in each spine type $(n=26$ dendrites from 7 mice). oneway ANOVA, $F(3,100)=7.17,{ }^{* \star *} p<0.001$.

(G) Spine survival fraction across processes $(n=26$ dendrites from 7 mice) recorded over 10 consecutive days.

(H) Percent of spines added between days over 10 days of consecutive imaging.

(I) Percent of spines subtracted between days over 10 days of consecutive imaging. 
bioRxiv preprint doi: https://doi.org/10.1101/2021.08.10.455885; this version posted November 8, 2021. The copyright holder for this preprint (which was not certified by peer review) is the author/funder, who has granted bioRxiv a license to display the preprint in perpetuity. It is made available under aCC-BY-NC 4.0 International license.

A

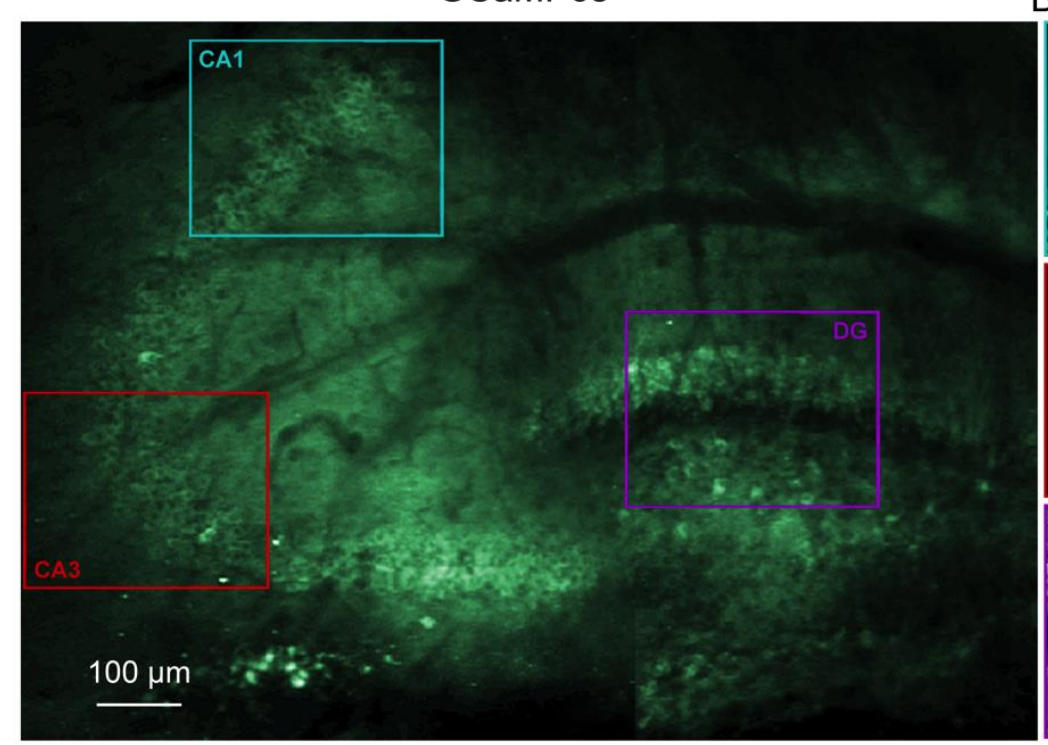

C

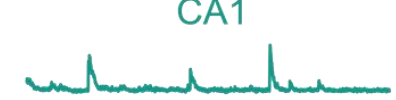

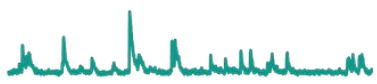

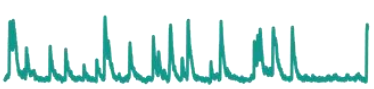

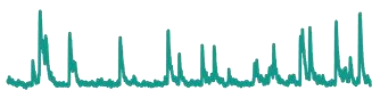
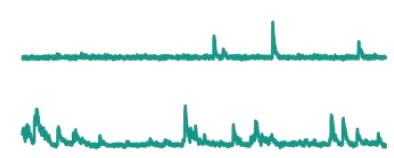
$200 \%$ L

$\mathrm{DF} / \mathrm{F} 25 \mathrm{~s}$

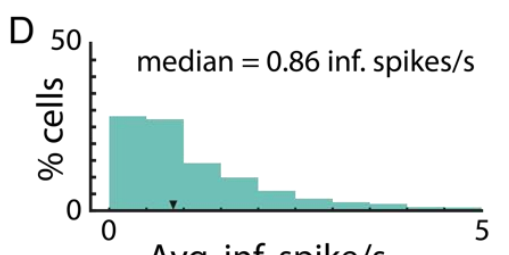

Avg. inf. spike/s.

E

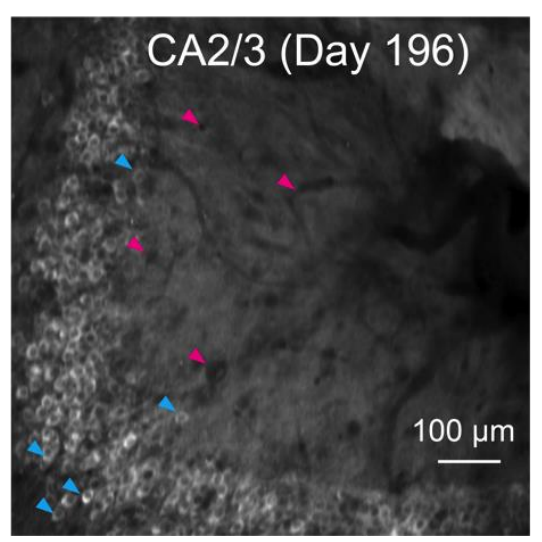

DF/F $25 \mathrm{~s}$
$100 \%$ L

DF/F $25 \mathrm{~s}$

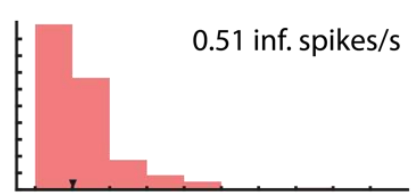

B

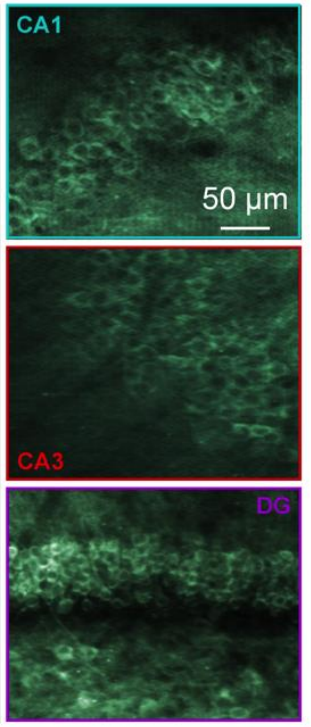

CA3
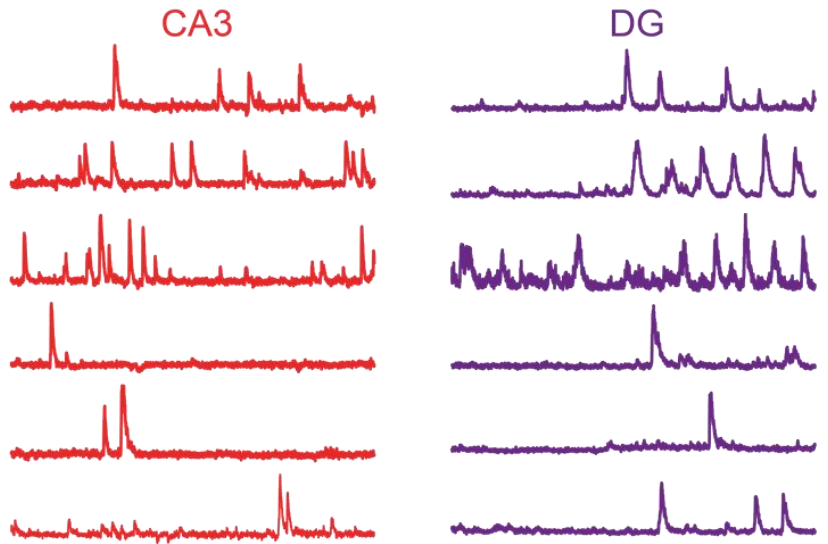

200\%L L

0.59 inf. spikes/s
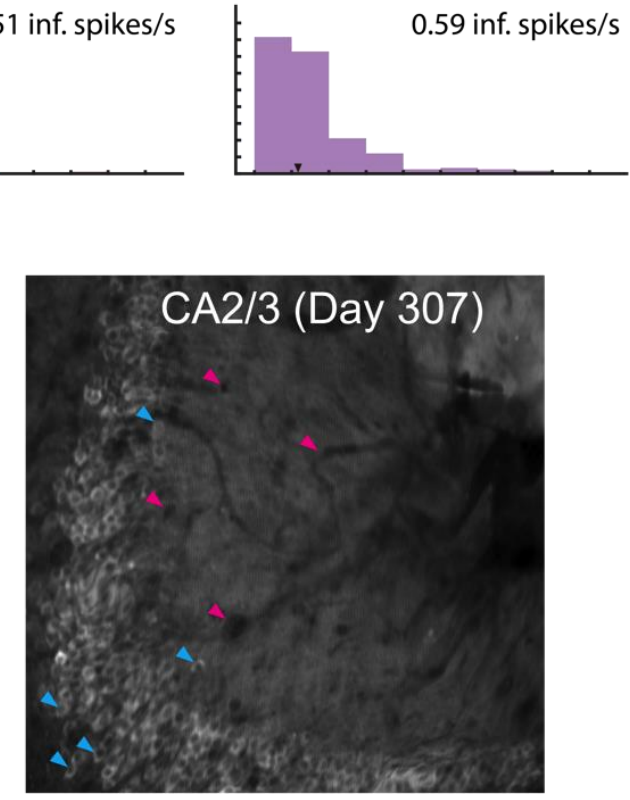
Figure 4. Microperiscope imaging of calcium dynamics across subfields in awake mice.

(A) Example tiled image (as in Fig. 1C) for a single panexcitatory transgenic GCaMP6s mouse (Slc17a7-GCaMP6s). Scale bar $=100 \mu \mathrm{m}$.

(B) Enlarged images of each hippocampal subfield (CA1, CA3, DG) corresponding to the rectangles in (A). Scale bar $=50 \mu \mathrm{m}$.

(C) Example GCaMP6s normalized fluorescence time courses (\% DF/F) for identified cells in each subfield.

(D) Distribution of average inferred spiking rate during running for each hippocampal subfield (CA1, CA3, DG). Median is marked by black arrowhead.

(E) Example average projection of CA2/3 imaging plane 196 days post implantation (left) and 111 days later. Image was aligned using non-rigid registration (see Methods) to account for small tissue movements. Magenta arrowheads mark example vasculature and blue arrowheads mark example neurons that are visible in both images. Scale bar $=100 \mu \mathrm{m}$. 
A

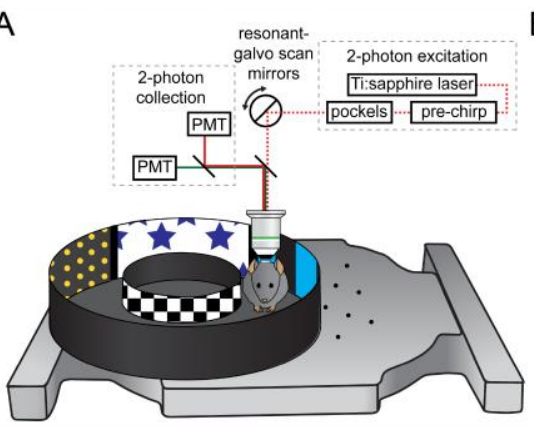

B

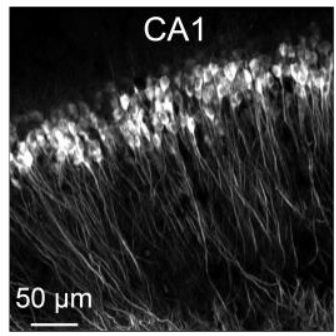

Maximum projections
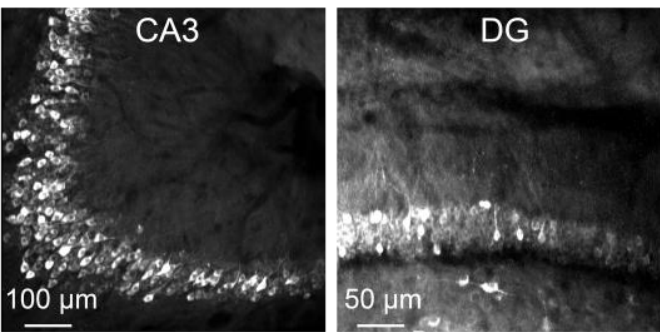

DG

$$
\text { c }
$$

CAI

СА

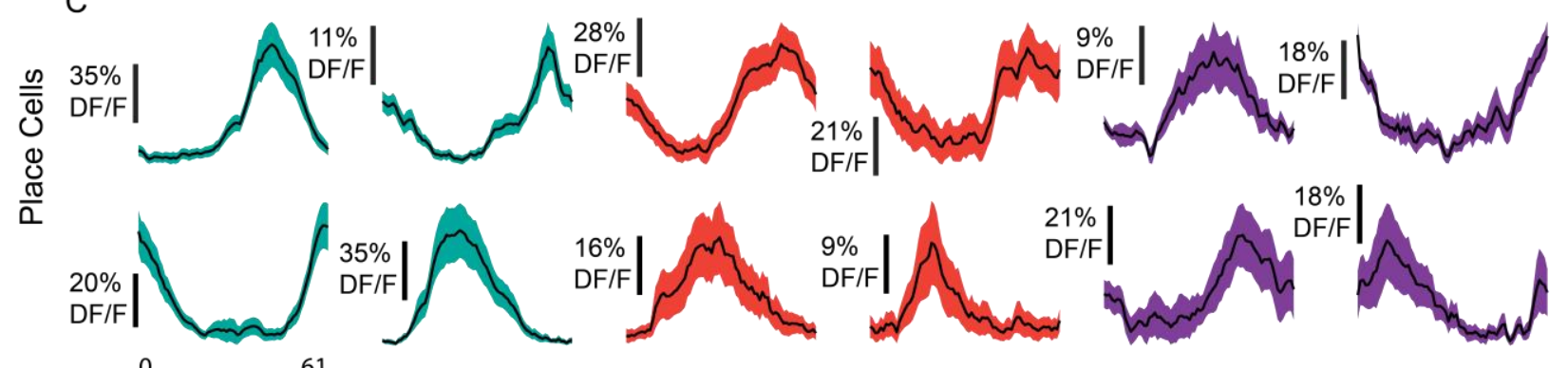

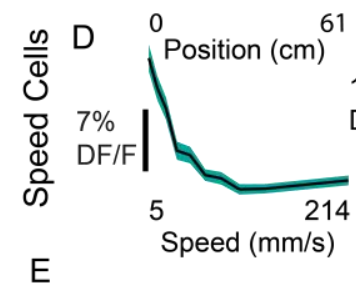

$\mathrm{E}$

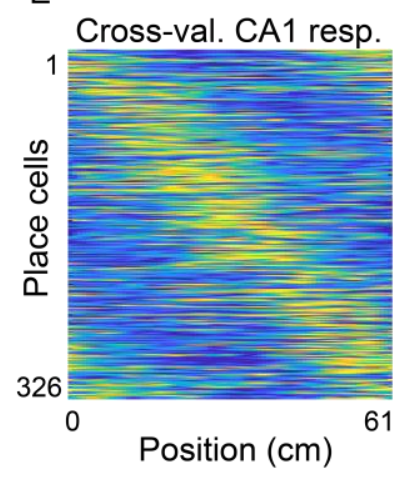

$\mathrm{F} \quad \mathrm{CA} 1(\mathrm{n}=1026)$

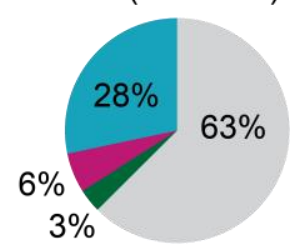

Place cells
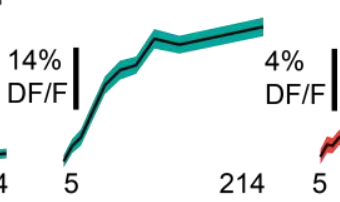

214
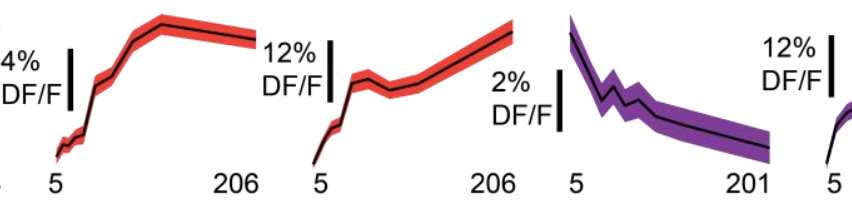

204

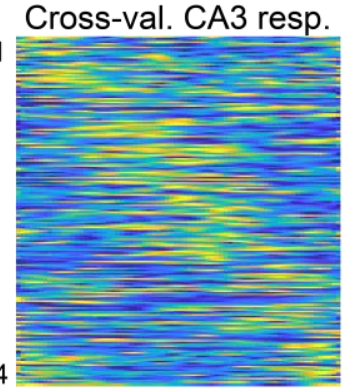

82

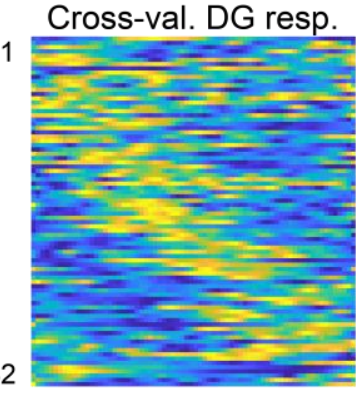

$0 \quad$ Normalized DF/F 1.0

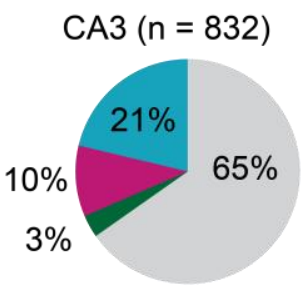

Speed cells Neither
$D G(n=463)$

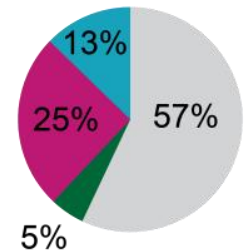

Conjunctive
G
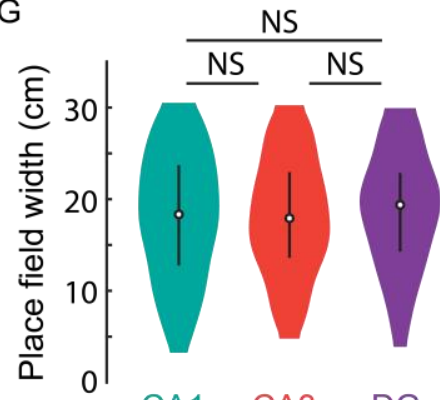

CA1 CA3 DG

Place cells only
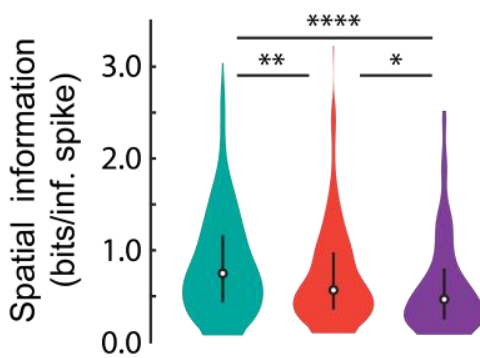

CA1 CA3 DG 
Figure 5. Prevalence of place and speed cells across hippocampal subfields.

(A) Schematic of air-lifted carbon fiber circular track (250 mm outer diameter) that the mice explored during imaging (Video S4). Four sections of matched visual cues lined the inner and outer walls.

(B) Example maximum projections of GCaMP6s-expressing neurons in each subfield. Scale bar $=50,100,50 \mu \mathrm{m}$ respectively.

(C) Plots of mean normalized calcium response (\% DF/F) versus position along the circular track for four identified place cells (PCs) in each subfield. Shaded area is s.e.m.

(D) Plots of mean calcium response (\% DF/F) versus speed along the circular track for two identified speed cells (SCs) in each subfield. Shaded area is s.e.m.

(E) Cross-validated average responses (normalized DF/F) of all PCs found in each subfield, sorted by the location of their maximum activity. Responses are plotted for even trials based on peak position determined on odd trials to avoid spurious alignment.

(F) Distribution of cells that were identified as PCs, SCs, conjunctive PC+SCs, and non-coding in each subfield.

(G) Distribution of place field width for all place cells in each subfield. Error bars indicate the interquartile range (75th percentile minus 25th percentile). Two-sample KolmogorovSmirnov test: CA1-CA3, $p=0.47$; CA3-DG, $p=0.21$; CA1-DG, $p=0.58$. NS, not significant $(p>0.05)$.

(H) Distribution of spatial information (bits per event) for all place cells in each subfield. Error bars are the same as in $(\mathrm{G})$. Two-sample Kolmogorov-Smirnov test: CA1-CA3, $p=0.0087$; CA3-DG, $p=0.04$; CA1-DG, $p=7.6 \times 10^{-5} .{ }^{*} p<0.05,{ }^{* *} p<0.01,{ }^{* \star *} p<0.0001$. 
A

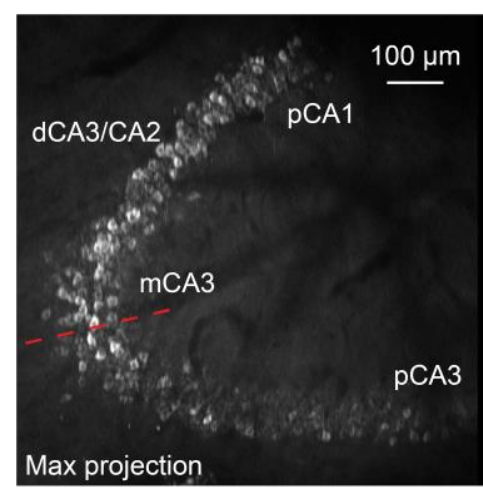

B

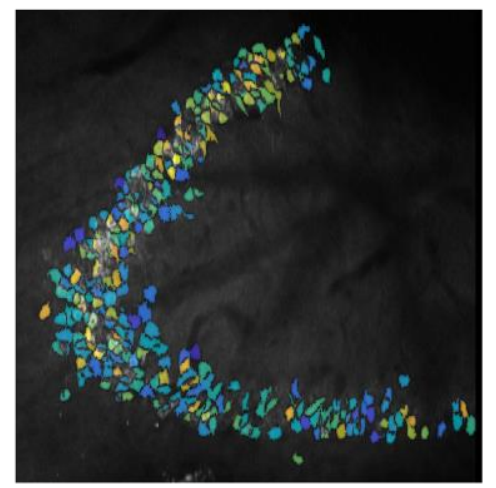

0.08 bits/inf. spike
C All cells $(n=1054)$

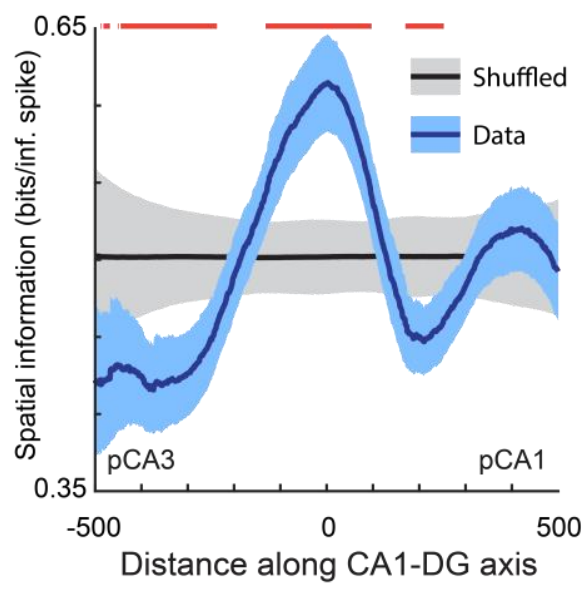

Figure 6. Spatial selectivity of neurons varies along the DG-to-CA1 axis.

(A) Maximum projection of an example DG-to-CA1 axis recording. Approximate locations of CA3 and CA1 subfields are labeled. Inflection point labeled with red line. Scale bar $=100$ $\mu \mathrm{m}$.

(B) Spatial information (bits/inferred spike) pseudo-colored on a logarithmic scale, for each neuron overlaid on the maximum projection in $(A)$.

(C) Spatial information as a function of distance along the DG-to-CA1 axis (pCA3 to dCA1) calculated with a sliding window; mean \pm bootstrapped s.e.m.; real data (blue), shuffled control (black). Red lines indicate values that are outside the shuffled distribution $(p<0.05)$. A general linear F-test revealed significant non-uniformity: $F(6,1030)=4.35, p=2.4 \times 10^{-4}$. 


\section{SUPPLEMENTAL FIGURES}

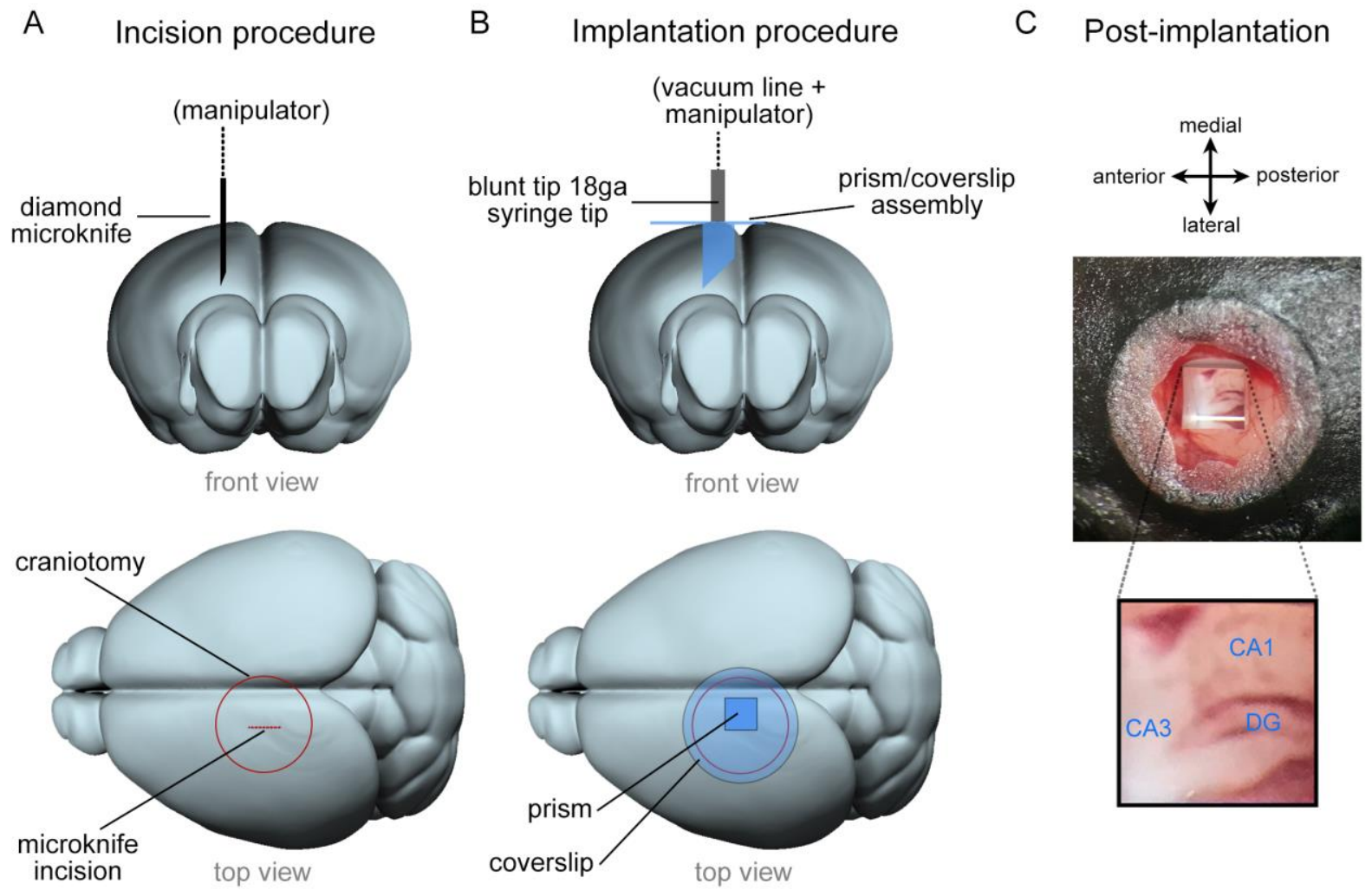

Figure S1: Overview of microperiscope implantation surgery.

(A) Schematic view from front (top row) and top (bottom row) of mouse brain indicating placement of the incision. The incision is made using the diamond microknife prior to the implantation of microperiscope. The microknife bevel faces medial to leave a flat surface for the microperiscope face.

(B) Schematic view from front (top row) and top (bottom row) of the brain during microperiscope implantation. The microperiscope is attached to the coverglass prior to implantation using optical glue, and the assembly is lowered into the incision using a blunt 18 gauge syringe mounted on a manipulator and held using a vacuum line. Once the assembly has been cemented to the skull, the vacuum is released and the manipulator removed. The microperiscope faces lateral for imaging of the transverse plane of the dorsomedial hippocampus.

(C) Post implantation view of microperiscope assembly, with identified hippocampal subregions. 
A

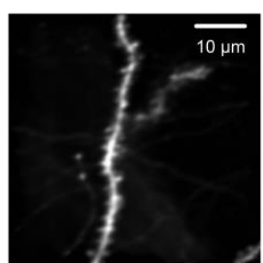

Mean Image

B

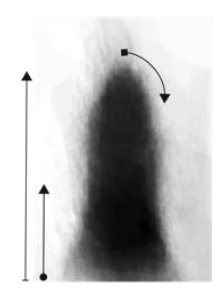

Filopodium

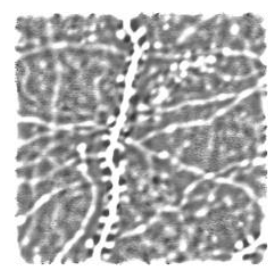

High-Pass

Filtered Image

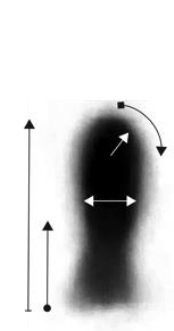

Mushroom

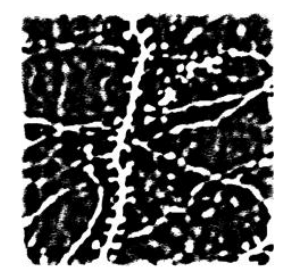

Binarization

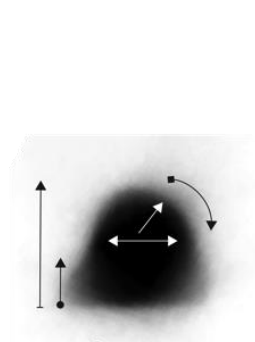

Stubby

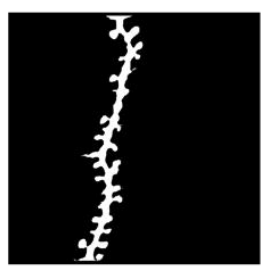

Morphological

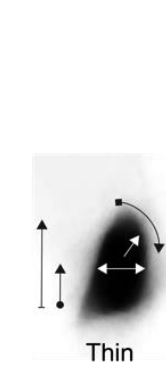

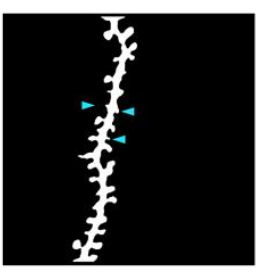

Semi-Automated Manual Curation

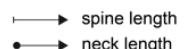

k length $\longrightarrow$ head circularity $\longrightarrow$ aspect ratio

Figure S2: Dendritic morphology image processing pipeline and spine classification.

(A) Steps in dendritic morphology image processing pipeline (see Methods). Weighted mean image is obtained by acquiring average projections from 4 planes spaced by $3 \mu \mathrm{m}$ and weighting the images around the brightest part of the dendrite. Next, the mean image is high-pass filtered to reveal fine structures. The image is then binarized using a global threshold, to filter out less prominent dendrites. Morphological opening is applied to remove any elements of the high-pass filtered image that survive the binarization process that are too small to be the principal dendrite. A semi-automated manual curation process is then used to add back any individual spines that were lost during binarization and remove any unwanted dendritic segments. Cyan arrowheads indicate manually added spines. To isolate the spines, the image is skeletonized and structures that protruded laterally from the dendritic shaft and had a total area of $>1 \mu \mathrm{m}^{2}$ were identified as spines. Scale bar $=10 \mu \mathrm{m}$.

(B) Mean profile of all filopodium, mushroom, stubby, and thin spines ( $n=47,431,695$, and 495 , respectively), computed by averaging across the registered spines of each morphological classification. Spines were classified according to spine length, neck length, neck width, head circularity, and eccentricity (see Methods). 

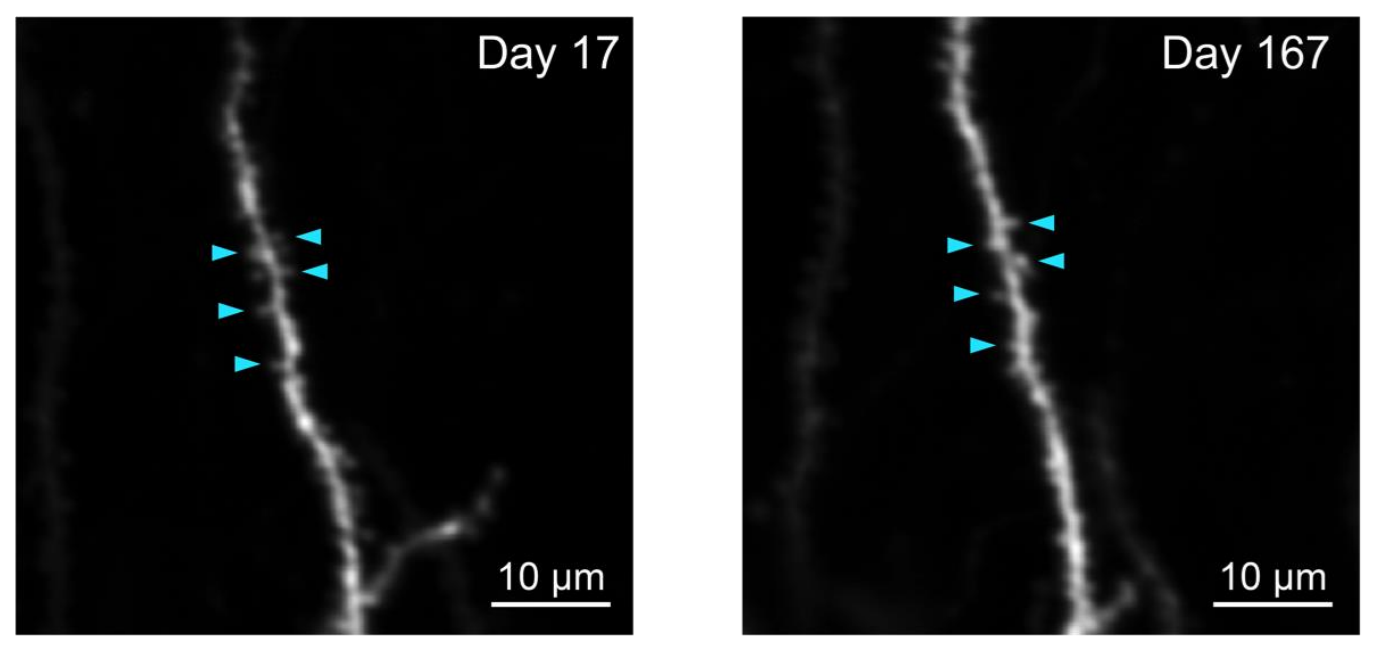

Figure S3: Long-term imaging of the same dendrite.

Weighted average projection of the same dendritic process 150 days apart. Spines present at both time points indicated by cyan arrowheads. Scale bar $=10 \mu \mathrm{m}$. 


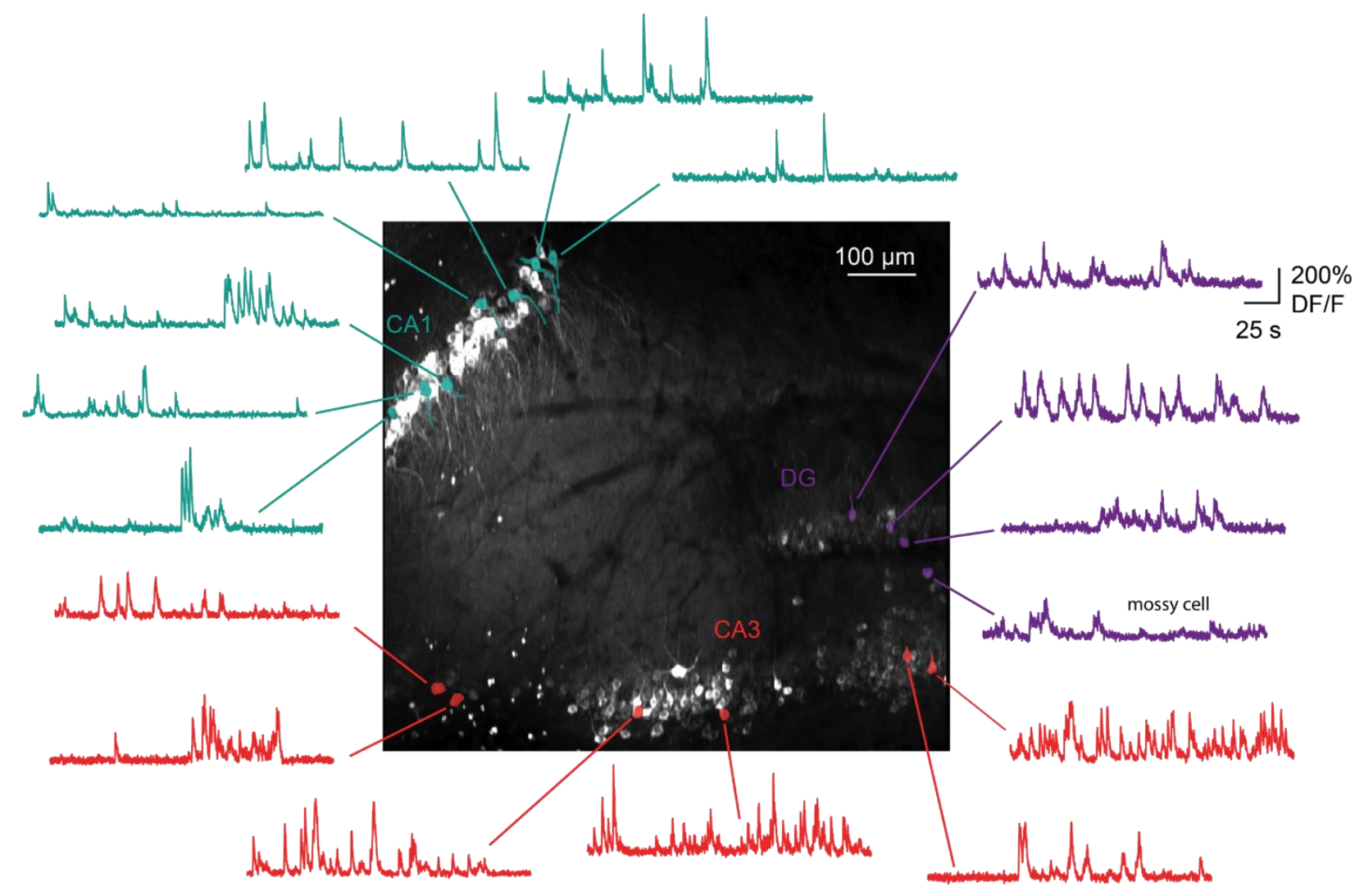

Figure S4. Simultaneous imaging of all three hippocampal subfields.

Maximum projection, and example GCaMP6s fluorescence time courses for identified cells, from a recording in which CA1 ( $n=55$ cells), CA3 $(n=158)$, and DG $(n=28)$ were simultaneously recorded from the same image plane. Scale bar $=100 \mu \mathrm{m}$. 
A
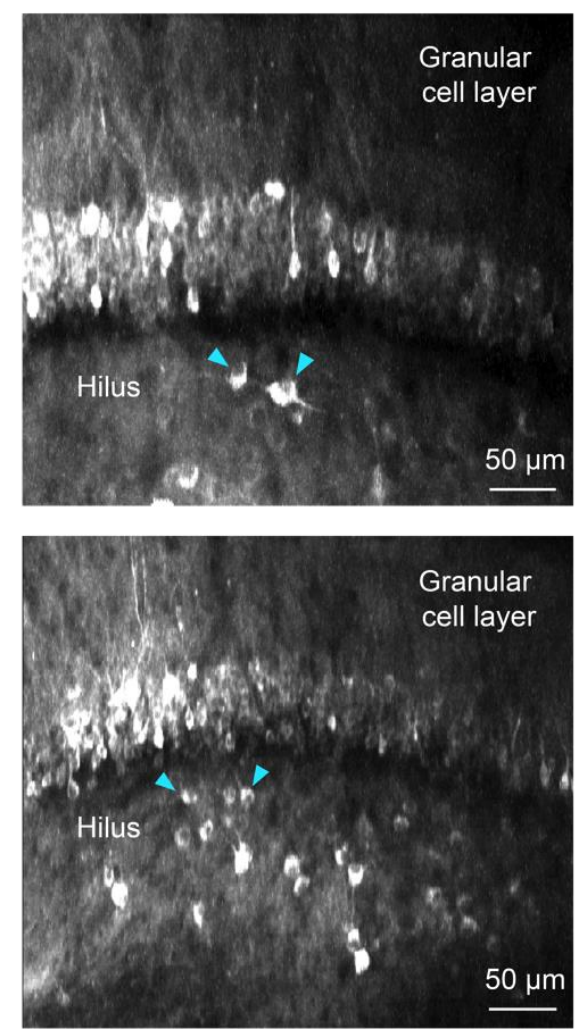

B

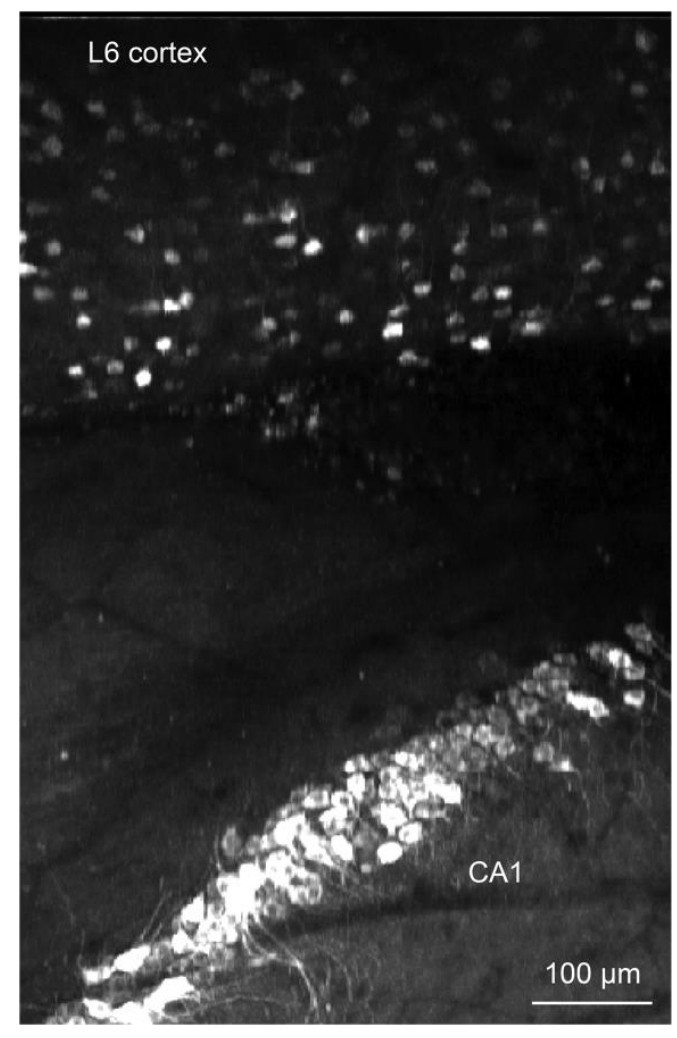

Figure S5. Additional applications for microperiscope imaging.

(A) Two maximum projections of imaging planes in which both the granular cell layer and the hilus in the DG could be imaged simultaneously. Example putative mossy cells indicated with cyan arrowheads. Scale bar $=50 \mu \mathrm{m}$.

(B) Maximum projection from an imaging session in which both CA1 and L6 of the neocortex are visible, allowing simultaneous imaging of neurons in CA1 and deep layers of the parietal cortex. Scale bar $=100 \mu \mathrm{m}$. 
A

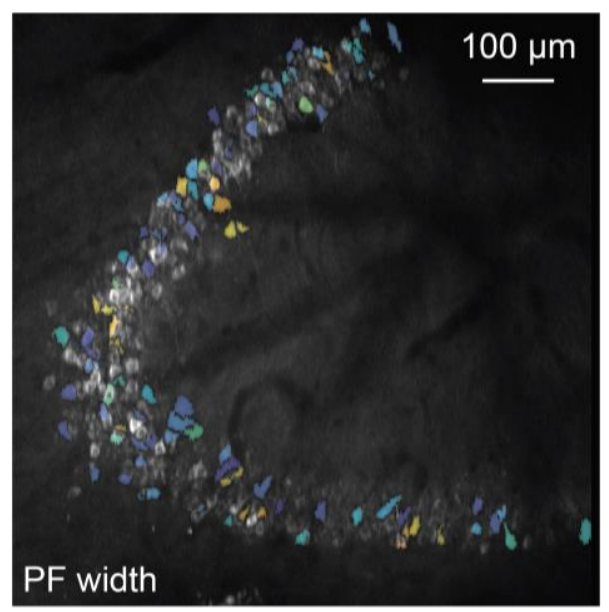

$4.4 \mathrm{~cm}$
B Place cells only $(n=248)$

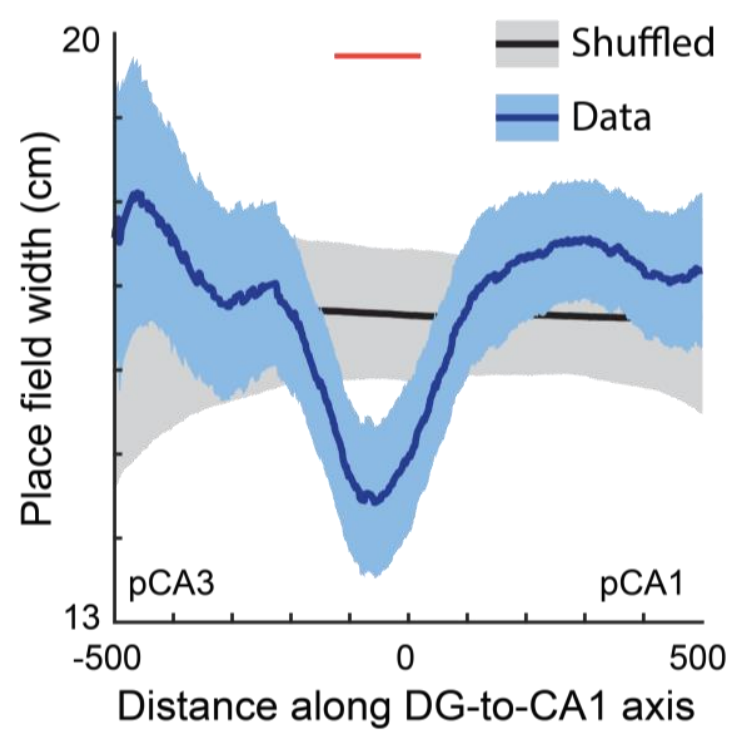

Figure S6. Place field width along the DG-to-CA1 axis.

(A) Place field width pseudo-colored for each place cell overlaid on the maximum projection from Fig. 5A. Any cell that was not a place cell is not pseudo-colored. Scale bar $=100 \mu \mathrm{m}$.

(B) Place field width, as a function of distance along the DG-to-CA1 axis (pCA3 to pCA1), of real data (blue) vs. shuffled control (black). Shaded area is s.e.m. Red lines indicate individual positions with place field values that were outside of the shuffled distribution $(p<$ $0.05)$. However, a General Linear F-test revealed no significant non-uniformity: $F(4,236)=$ $1.28, p=0.28$. 


\section{SUPPLEMENTAL VIDEOS}

Video S1. Demonstration of two-photon imaging of a Slc17a7-GCaMP6s mouse through the microperiscope. Recording starts in the superficial cortex in front of microperiscope, then moves through the microperiscope to the hippocampus, zooming in on subfields CA1, CA3, and DG.

Video S2. Calcium activity in subfield CA1 in a Slc17a7-GCaMP6s mouse imaged through the microperiscope.

Video S3. Calcium activity in subfield DG in a Slc17a7-GCaMP6s mouse imaged through the microperiscope.

Video S4. Mouse running in the floating chamber circular track. Ambient light is higher than usual levels for improved video quality. 


\section{METHODS}

\section{Animals}

For dendritic morphology experiments, Thy1-GFP-M (Jax Stock \#007788) transgenic mice ( $n=$ 7) were used for sparse expression of GFP throughout the forebrain. For forebrain-wide calcium indicator expression, Emx1-IRES-Cre (Jax Stock \#005628) x ROSA-LNL-tTA (Jax Stock \#011008) x TITL-GCaMP6s (Jax Stock \#024104) triple transgenic mice $(n=2)$ or Slc17a7IRES2-Cre (Jax Stock \#023527) x TITL2-GC6s-ICL-TTA2 (Jax Stock \#031562) double transgenic mice $(n=6)$ were bred to express GCaMP6s in excitatory neurons. For imaging experiments, 8-51 week old (median 17 weeks) mice of both sexes (6 males and 9 females) were implanted with a head plate and cranial window and imaged starting 2 weeks after recovery from surgical procedures and up to 10 months after microperiscope implantation. The animals were housed on a $12 \mathrm{hr}$ light/dark cycle in cages of up to 5 animals before the implants, and individually after the implants. All animal procedures were approved by the Institutional Animal Care and Use Committee at University of California, Santa Barbara.

\section{Surgical Procedures}

All surgeries were conducted under isoflurane anesthesia (3.5\% induction, $1.5-2.5 \%$ maintenance). Prior to incision, the scalp was infiltrated with lidocaine (5 mg kg-1, subcutaneous) for analgesia and meloxicam ( $2 \mathrm{mg} \mathrm{kg}-1$, subcutaneous) was administered preoperatively to reduce inflammation. Once anesthetized, the scalp overlying the dorsal skull was sanitized and removed. The periosteum was removed with a scalpel and the skull was abraded with a drill burr to improve adhesion of dental acrylic.

For hippocampal imaging, we used two types of custom-designed glass microperiscope (Tower Optical). The first ( $\mathrm{v} 1_{\mathrm{CA} 1}$ ), for imaging the upper part of the hippocampus (CA1/CA2) consisted of a $1 \times 1 \times 1 \mathrm{~mm}$ square base and a $1 \mathrm{~mm}$ right angle prism, for a total length of 2 $\mathrm{mm}$ on the longest side (Fig. 1B, left). The second ( $\mathrm{V} 2_{\mathrm{HPC}}$ ), for imaging the entire transverse circuit (CA1-3, DG) had a $1.5 \times 1.5 \times 1.0 \mathrm{~mm}(\mathrm{~L} \times \mathrm{W} \times \mathrm{H})$ square base and a $1.5 \mathrm{~mm}$ right angle prism, for a total length of $2.5 \mathrm{~mm}$ on the longest side (Fig. 1B, right). The hypotenuse of the right angle prisms were coated with enhanced aluminum for internal reflectance. The microprism was attached to a $5 \mathrm{~mm}$ diameter coverglass (Warner Instruments) with a UV-cured optical adhesive (Norland, NOA61). Prior to implantation, the skull was soaked in sterile saline and the cortical vasculature was inspected to ensure that no major blood vessels crossed the incision site. If the cortical vasculature was suitable, a 3-4 $\mathrm{mm}$ craniotomy was made over the implantation site (centered at $2.2 \mathrm{~mm}$ posterior, 1.2-1.7 mm lateral to Bregma). For the smaller 
microperiscope ( $\mathrm{v} 1_{\mathrm{CA} 1}$ ), a $1 \mathrm{~mm}$ length anterior-to-posterior incision centered at $-2.1 \mathrm{~mm}$ posterior, $1.2 \mathrm{~mm}$ lateral to Bregma was then made through the dura, cortex, and mediodorsal tip of the hippocampus to a depth of $2.2 \mathrm{~mm}$ from the pial surface with a sterilized diamond micro knife (Fine Science Tools, \#10100-30) mounted on a manipulator (Fig. S1A). For the larger microperiscope (v2 $\mathrm{HPC}$ ), two overlapping $1.0 \mathrm{~mm}$ length anterior-to-posterior incisions were made centered at $-1.8 \mathrm{~mm}$ posterior / $1.7 \mathrm{~mm}$ lateral and $-2.4 \mathrm{~mm}$ posterior $/ 1.7 \mathrm{~mm}$ lateral to Bregma to a depth of $2.7 \mathrm{~mm}$, with a total anterior-to-posterior incision length of 1.6 $\mathrm{mm}$. Note that placements in the regions shown in Fig. 1B required incision coordinates slightly posterior to those indicated on the atlas. Care was taken not to sever any major cortical blood vessels. Gelfoam (VWR) soaked in sterile saline was used to remove any blood from the incision site. Once the incision site had no bleeding, the craniotomy was submerged in cold sterile saline, and the microprism was lowered into the cortex using a manipulator, with the imaging face of the prism facing lateral (Fig. S1B). Once the microprism assembly was completely lowered through the incision until the coverglass was flush with the skull, the edges of the window were sealed with silicon elastomer (Kwik-Sil, World Precision Instruments), then with dental acrylic (C\&B-Metabond, Parkell) mixed with black ink (Fig. S1C). Care was taken that the dental cement did not protrude over the window, as it could potentially scratch the objective lens surface. Given the working distance of the objective used in this study ( $3 \mathrm{~mm})$, the smaller microperiscope ( $\mathrm{v} 1_{\mathrm{CA} 1}$ ) implant enabled imaging from 2250 - $2600 \mu \mathrm{m}$ below the coverglass surface, corresponding to approximately 150 - $500 \mu \mathrm{m}$ into the lateral hippocampus (the $150 \mu \mathrm{m}$ of tissue nearest to the prism face was not used for imaging). The larger microperiscope (v2 $\mathrm{HPC}$ ) implant enabled imaging from 2650 - $2850 \mu \mathrm{m}$ below the coverglass surface, corresponding to approximately 150 - $350 \mu \mathrm{m}$ into the lateral hippocampus (the $150 \mu \mathrm{m}$

407 of tissue nearest to the prism face was not used for imaging). The microprism implantations were stable for up to ten months following the surgery (Fig. 4E).

After microperiscope implantation, a custom-designed stainless steel head plate

411 black ink. After surgery, mice were administered carprofen (5 - $10 \mathrm{mg} \mathrm{kg}-1$, oral) every $24 \mathrm{hr}$ for

4123 days to reduce inflammation. Microperiscope designs and head fixation hardware are

413 available on our institutional lab web site (https://goard.mcdb.ucsb.edu/resources).

\section{Point Spread Function Measurements}

415 To measure empirical point spread functions, fluorescent microspheres $(0.2 \mu \mathrm{m}$ yellow-green

416 fluorescent microspheres; ThermoFisher F8811) were embedded 1:2000 in 0.5\% agar and

417 placed under the cranial window or on the face of the microperiscope. Image stacks were taken 
418 through the microspheres $(0.06 \mu \mathrm{m}$ per pixel in $\mathrm{XY} ; 0.5 \mu \mathrm{m}$ per plane in $\mathrm{Z})$, and candidate

419 microspheres were isolated using a watershed algorithm (Matlab image processing toolbox).

420 Only microspheres that were $>20$ pixels $(1.2 \mu \mathrm{m})$ away from nearest neighbor microspheres and

421 completely contained within the Z-stack were used for further analysis. We registered isolated

422 microspheres at their centroids and measured the full width at half maximum (FWHM) of the

423 average $X Y$ and $X Z$ profiles to determine the lateral and axial resolution, respectively.

424 Since the geometry of the microperiscope limits the angle of the focusing light cone

425 through the microperiscope, it predominately determines the functional numerical aperture at

426 the imaging plane. Based on the microperiscope geometry, we calculated the effective NA of

427 the v1 $1_{\mathrm{CA} 1}$ microperiscope and $\mathrm{v} 2_{\mathrm{HPC}}$, and used it to calculate the theoretical lateral and axial

428 resolution according to the following formulae ${ }^{89}$

$$
\omega_{X Y}= \begin{cases}\frac{0.320 \lambda}{\sqrt{2} N A} & N A<0.7 \\ \frac{0.325 \lambda}{\sqrt{2} N A^{0.91}} & N A>0.7\end{cases}
$$

$$
\omega_{Z}=\frac{0.532 \lambda}{\sqrt{2}}\left[\frac{1}{n-\sqrt{n^{2}-N A^{2}}}\right]
$$

To perform aberration correction with adaptive optics, a deformable mirror (Multi-3.5, Boston Micromachines Corporation) was set at a plane conjugate to the raster scanning mirrors and the back aperture of the objective lens in the two-photon imaging system. Fluorescent microspheres $(0.2 \mu \mathrm{m})$ were imaged, and the standard deviation of the image brightness was maximized under different configurations of the deformable mirror. Twelve selected Zernike modes are applied and modulated sequentially over a total of three rounds. The 12 zernike modes are: 1) oblique astigmatism, 2) vertical astigmatism, 3) vertical trefoil, 4) vertical coma, 5) horizontal coma, 6) oblique trefoil, 7) oblique quadrafoil, 8) oblique secondary astigmatism, 9) primary spherical, 10) vertical secondary astigmatism, 11) vertical quadrafoil, 12) secondary spherical. For each Zernike mode, 21 steps of amplitudes were scanned through, and images were acquired for each step. The amplitude that resulted in the largest standard deviation was saved and set as the starting point of the DM configuration for the scanning of the next Zernike mode. The brightness, the lateral resolution, and the axial resolution are compared with and

444 without the application of the deformable mirror correction. 
447 chamber $^{34}$ (Mobile Homecage, NeuroTar, Ltd). The chamber base was embedded with magnets

448 to allow continual tracking of the position and angular displacement of the chamber. Behavioral

449 data was collected via the Mobile HomeCage motion tracking software (NeuroTar, versions

450 2.2.0.9, 2.2.014, and 2.2.1.0 beta 1). During imaging experiments, image acquisition was

451 triggered using a TTL pulse from the behavioral software to synchronize the timestamps from

452 the $2 \mathrm{P}$ imaging and chamber tracking.

453 A custom carbon fiber arena (250 $\mathrm{mm}$ diameter) was lined with four distinct visual

454 patterns (5.7 cm tall, $18.1 \mathrm{~cm}$ wide) printed on 7 mil waterproof paper (TerraSlate) with black

455 rectangles $(5.7 \mathrm{~cm}$ tall and $1.5 \mathrm{~cm}$ wide) placed in between the four patterns. A circular track

456 (Fig. 5A; Video S4) was made by adding a removable inner circle (14 cm in diameter and 4.2

$457 \mathrm{~cm}$ tall) with visual cues that were matched to the outer wall printed on 7 mil waterproof paper.

458 Transparent tactile stickers (Dragon Grips) were placed on the arena floors to give differential

459 tactile stimuli along the track. In between each recording and or behavioral session, the arena

460 walls and floors were thoroughly cleaned.

$461 \quad$ Mice were acclimated to the arena by the following steps: 1) On the first day the mice

462 were placed into the chamber and allowed to freely explore without head fixation for 15 - 20

463 minutes. A piece of plexiglass with holes drilled through was placed on top of the arena to keep

464 the mice from climbing out. 2) On the second day, the mice were head-fixed to a crossbar

465 extending over the floating chamber (Fig. 5A) and allowed to freely explore the floating chamber

466 freely for 15 minutes. Air flow (3 - 6.5 psi) was adjusted to maximize steady walking/running. On

467 subsequent days, the head fixation time was increased by increments of 5 minutes, as long as

468 the mice showed increased distance walked and percent time moving. This was continued until

469 the mice would explore for 30 - 40 minutes and run for greater than 15\% of the time. 3) Mice

470 were head-fixed in the floated chamber for 20 minutes with a custom light blocker attached to

471 their headplate. 4) Mice were head-fixed and placed on the 2P microscope to allow habituation

472 to the microscope noise. 5) After mice were fully habituated, 20 - 40 minute duration recording

473 sessions on the $2 \mathrm{P}$ microscope were performed.

474 If at any point during the above acclimation protocol the mouse significantly decreased

475 distance traveled or percentage of time moving, then the mouse was moved back to the

476 previous step.

477 Custom software was written to process the behavioral data output by the Mobile

478 HomeCage motion tracking software. Because the Mobile HomeCage motion tracking software

479 sampling rate was faster than the frame rate of our 2P imaging, all behavioral variables (speed, 
location, polar coordinates, and heading) that were captured within the acquisition of a single 2P frame were grouped together and their median value was used in future analysis. For the polar angle (which we used as the location of the mouse in 1D track), the median was computed using an open source circular statistics toolbox (CircStat 2012a) written for Matlab ${ }^{90}$. We removed any time points when the mouse was not moving, as is standard for measurement of place fields ${ }^{25}$. This helps separate processes that are related to navigation from those that are related to resting state. To do this, we smoothed the measured instantaneous speed and kept

487 time periods $>1 \mathrm{~s}$ that had speeds greater than $20 \mathrm{~mm} / \mathrm{s}$ (adding an additional $0.5 \mathrm{~s}$ buffer on 488 either side of each time period).

\section{Two-photon Imaging}

490 After recovery from surgery and behavioral acclimation, GFP or GCaMP6s fluorescence was 491 imaged using a Prairie Investigator 2P microscopy system with a resonant galvo scanning 492 module (Bruker). For fluorescence excitation, we used a Ti:Sapphire laser (Mai-Tai eHP, 493 Newport) with dispersion compensation (Deep See, Newport) tuned to $\lambda=920 \mathrm{~nm}$. Laser power 494 ranged from $40-80 \mathrm{~mW}$ at the sample depending on GCaMP6s expression levels.

495 Photobleaching was minimal $\left(<1 \% \mathrm{~min}^{-1}\right)$ for all laser powers used. For collection, we used 496 GaAsP photomultiplier tubes (H10770PA-40, Hamamatsu). A custom stainless-steel light blocker (eMachineShop.com) was mounted to the head plate and interlocked with a tube around the objective to prevent light from the environment from reaching the photomultiplier tubes. For imaging, we used a 16x/0.8 NA microscope objective (Nikon) to collect $760 \times 760$ pixel frames with field sizes of $829 \times 829 \mu \mathrm{m}$ or $415 \times 415 \mu \mathrm{m}$. Images were collected at $20 \mathrm{~Hz}$ and stored at $10 \mathrm{~Hz}$, averaging two scans for each image to reduce shot noise. aligned based on the average projection from a reference session, guided by stable structural

504 landmarks such as specific neurons and dendrites. Physical controls were used to ensure 505 precise placement of the head plate, and data acquisition settings were kept consistent across 506 sessions. Images were collected once every day for 5 - 10 days.

\section{Two-photon Post-processing}

508 Images were acquired using PrairieView acquisition software (Bruker) and converted into multi509 page TIF files. 
512 projections were weighted according to a Gaussian distribution across planes. Non-rigid

513 registration was used to align dendritic segments across consecutive recording sessions. The

514 registered images underwent high-pass filtering to extract low amplitude spine features using

515 code adapted from Suite2P's enhanced mean image function ${ }^{91}$ (Fig. S2A). The resulting ROls

516 were binarized using Otsu's global threshold method for spine classification (Fig. S2A). In most

517 cases, the global threshold successfully isolated the single most prominent dendrite. In fields

518 with higher background dendrites that were not desired, these extraneous dendrites were

519 manually excluded. To identify spines that fall below the global threshold, the user manually

520 specifies incrementally lower thresholds from which to select spines that were excluded in the

521 initial binarization. (Fig. S2A). Spines above the global threshold with an area of $>1 \mu \mathrm{m}^{2}$ were

522 included in our analysis. To classify each spine as one of the four major morphological classes,

523 we performed the following steps. First, we found the base of the spine by identifying the region

524 closest to the dendritic shaft. Second, we calculated the length of the spine by taking the

525 Euclidean distance between the midpoint of the spine base and the most distant pixel. Third,

526 this vector was divided evenly into three segments to find the spine head, neck, and base areas

527 respectively. Finally, spines were classified in the four categories, considering the following

528 threshold parameters (Fig. S2B): stubby (neck length $<0.2 \mu \mathrm{m}$ and aspect ratio $<1.3$ ), thin

529 (neck length $>0.2 \mu \mathrm{m}$, spine length $<0.7 \mu \mathrm{m}$, head circularity $<0.8 \mu \mathrm{m}$ ), mushroom (neck

530 length $>0.2 \mu \mathrm{m}$, head circularity $>0.8 \mu \mathrm{m}$ ), and filopodium (neck length $>0.2 \mu \mathrm{m}$, spine length

$531<0.8 \mu \mathrm{m}$, aspect ratio $>1.3)$.

532 For calcium imaging sessions, the TIF files were processed using the Python

533 implementation of Suite2 $\mathrm{P}^{91}$. We briefly summarize their pipeline here. First, TIFs in the image

534 stack undergo rigid registration using regularized phase correlations. This involves spatial

535 whitening and then cross-correlating frames. Next, regions of interest (ROls) are extracted by

536 clustering correlated pixels, where a low-dimensional decomposition is used to reduce the size

537 of the data. The number of ROls is set automatically from a threshold set on the pixel

538 correlations. We manually checked assigned ROls based on location, morphology and DF/F

539 traces.

Since the hippocampal pyramidal cells are densely packed and the prism reduces the

541 axial resolution, we perform local neuropil subtraction using custom code

542 (https://github.com/ucsb-goard-lab/two-photon-calcium-post-processing) to avoid neuropil

543 contamination. The corrected fluorescence was estimated according to

$$
F_{\text {corrected }}(n)=F_{\text {soma }}(n)-\alpha\left(F_{\text {neuropil }}(n)-\bar{F}_{\text {neuropil }}\right) \text {, }
$$


where $F_{\text {neuropil }}$ was defined as the fluorescence in the region $<30 \mu \mathrm{m}$ from the ROI border (excluding other ROIs) for frame $n . \bar{F}_{\text {neuropil }}$ is $F_{\text {neuropil }}$ averaged over all frames. $\alpha$ was chosen from $[0,1]$ to minimize the Pearson's correlation coefficient between $F_{\text {corrected }}$ and $F_{\text {neuropil. }}$. The $\Delta \mathrm{F} / \mathrm{F}$ for each neuron was then calculated as

$$
\frac{\Delta F}{F}=\frac{F_{n}-F_{0}}{F_{0}},
$$

where $F_{n}$ is the corrected fluorescence $\left(F_{\text {corrected }}\right)$ for frame $n$ and $F_{0}$ is defined as the first mode of the corrected fluorescence density distribution across the entire time series.

We deconvolved this neuropil subtracted $\Delta \mathrm{F} / \mathrm{F}$ to obtain an estimate for the instantaneous spike rate, which we used (only) for the computation of neurons' spatial information (see below). This inferred spike rate was obtained via a MATLAB implementation of a sparse, nonnegative deconvolution algorithm (OASIS) used for $\mathrm{Ca}^{2+}$ recordings ${ }^{92}$. We used an

557 auto-regressive model of order 1 for the convolution kernel.

\section{Spine Imaging Data Analysis}

559 After nonrigid registration, high pass filtering, and binarization of the dendritic segment, individual spines were extracted based on standard morphological criteria ${ }^{93}$. Spines projecting laterally from the dendritic segment were extracted and analyzed as individual objects, as described previously (Fig. S2). The sum of the members of each spine class, as well as the total number of all spines, was recorded for each session. Spine totals ( $\left.S_{\text {total }}\right)$ were then broken down into $10 \mu \mathrm{m}$ sections of the dendritic segment $\left(S_{\text {section }}\right)$ using the following calculation: where length of the dendritic segment, $D_{\text {length }}$, was determined by skeletonizing the dendritic shaft to 1 pixel in diameter, then taking the area of the pixels. $F_{\text {pixels }}$ is the FOV in pixels, which

$$
S_{\text {section }}(n)=\frac{S_{\text {total }}(n)}{\left(D_{\text {length }} * \frac{F_{\mu m}}{F_{\text {pixels }}}\right)} * 10 \text {, }
$$
here was $760 \times 760$ at $16 \times$ magnification, and $F_{\mu m}$ is the FOV in microns, which was $52 \times 52 \mu \mathrm{m}$. Turnover was estimated at $24 \mathrm{~h}$ increments; turnover here is defined as the net change

572 were involved in turnover across days, segments recorded $24 \mathrm{~h}$ apart were aligned and overlaid 
573 using a custom MATLAB interface, which allowed the user to manually select new or removed

574 spines. Percent addition/subtraction $S_{a / s}$ was calculated as:

575

576

577

578

579

580

581

582

583

584

585

586

587

588

589

590

591

592

593

594

595

596

597

598

599

600

601

602

$$
S_{a / s}=\frac{N_{a / s}(t)}{N(t)} * 100
$$

where $N_{a / s}(t)$ is spines that have been added or subtracted and $N(t)$ is the total average number of spines. To account for variance in spine classification across days, turnover of specific classes of spines was normalized to total cumulative turnover per day.

To calculate the survival fraction curve $S(t)$, we determined which spines were present at time $t_{n}$ that were not present at time $t_{0}^{43,44}$. The dendritic segment from $t_{0}$ was transparently overlaid with segments from $t_{n}$, and replacement spines that were present in $t_{0}$ but not $t_{n}$ were manually identified. Survival fraction was quantified as

$$
S(t)=\frac{N_{r}\left(t_{n}\right)}{N\left(t_{0}\right)} * 100
$$

where $N_{r}\left(t_{n}\right)$ are the total spines at $t_{n}$ that were also found in $t_{0}$, and $N\left(t_{0}\right)$ are the total number of spines that were present in $t_{0}$. Survival fraction, as well as \% addition and subtraction, was calculated in $10 \mu \mathrm{m}$ sections to control for segment length.

\section{Calcium Imaging Data Analysis}

For calcium imaging experiments during exploration of the air-floated chamber, processed and synchronized behavioral data and $2 \mathrm{P}$ imaging data were used to identify place and speed cells as follows.

First, the 1D track was divided into 72 equal bins (each $\sim 0.85 \mathrm{~cm}$ in length). Activity as a function of position (we refer to these as spatial tuning curves) was computed for each lap, with activity divided by occupancy of each binned location. To avoid misattribution of slow calcium signals to spatial bins, any lap where the average instantaneous speed was greater than 180 $\mathrm{mm} / \mathrm{s}$, or where the total length of the lap took less than 1 second, were removed and not considered for further analysis. To assess the consistency of spatial coding of each cell, we randomly split the laps into two groups and computed the correlation coefficient between the averaged spatial tuning curves. We then did the same for shuffled data in which each lap's spatial tuning curve was circularly permuted by a random number of bins. This was performed 500 times, and the distribution of actual correlation coefficient values was compared to the 
603

distribution of circularly shuffled values using a two-sample Kolmogorov-Smirnov test $(\alpha=0.01)$. A cell that passed this test was considered a "consistent" cell.

To identify a neuron as a place cell, the neuron had to pass the consistency test, in addition to being well fit by a Gaussian function, $R_{D F / F}=A_{0}+A e^{\left(\frac{(X-B)}{C}\right)^{2}}$, with FWHM $=2 C \sqrt{\ln 2}$. Note that in this convention, $C^{2}=2 \sigma^{2}$. Specifically, we required that: 1 ) the adjusted $\mathrm{R}^{2}>$ $0.375 ; 2) 2.5 \mathrm{~cm}<F W H M<30.6 \mathrm{~cm}$ (50\% of track length); 3$) A>0 ; 4) A / A_{0}>0.50$. Cells that met these conditions were characterized as place cells; with place fields at the location of maximal activity and width defined as the FWHM.

Speed cells were identified using a standard process developed for identification of speed cells in medial entorhinal cortex and hippocampus ${ }^{62-64}$. We computed the Pearson correlation of each cells' DF/F trace with the mouse's speed across the experiment. This value is considered as a "speed score". We then circularly shuffled the DF/F 100 times (making sure that the amount shuffled was greater than 10 frames to ensure that the shuffled distribution did not have artificially high correlations). Cells whose speed score was greater than $99 \%$, or less than $1 \%$, of the shuffled distribution were considered speed cells.

To compute the spatial information ${ }^{94}$ of cell $j\left(S I_{j}\right)$, we used the following formula

$$
S I_{j}=\frac{1}{\bar{a}_{j}} \sum_{k=1}^{72} p(k) a_{j}(k) \log _{2}\left[\frac{a_{j}(k)}{\bar{a}_{j}}\right]
$$

where $\bar{a}_{j}$ is the mean inferred spike rate of cell $j, a_{j}(k)$ is the mean inferred spike rate of cell $j$ at position bin $k$, and $p(k)$ is the probability of being at position bin $k$. We divide by $\bar{a}_{j}$ to have $S I$ in units of bits/inferred spike.

To align recordings where we recorded along the CA1-DG axis, we found the inflection point of the axis and then computed the distance of each cell to that point. To do this, we performed the following steps. 1) We extracted the position of each identified cell using Suite2p's centroid output. 2) We then fit a function of the form $a(x-b)^{2}+c$ to the cell positions by rotating the field-of-view from 0 to 180 degrees and finding the rotation that maximized the $\mathrm{R}^{2}$ value of the fit. 3) We determined the inflection point as the peak of the curve and de-rotated the fit to determine the inflection point and curve in the original coordinates. The distance of each cell to the inflection point was found by finding the point along the fit curve that had the minimal distance to the cells centroid. 


\section{Immunohistochemistry}

633 Samples were perfusion fixed using 4\% paraformaldehyde in $0.1 \mathrm{M}$ sodium cacodylate buffer

$634(\mathrm{pH}=7.4)$ for 10 mins, and then immersion fixed overnight at $4^{\circ} \mathrm{C}$. Next, sections were rinsed in

635 cold PBS $5 \times 5$ mins and $1 \times 1 \mathrm{hr}$. Whole brains were then embedded in $10 \%$ low-melting

636 agarose. Subsequently, $100 \mu \mathrm{m}$ coronal sections were cut using a vibratome (Leica, Lumberton,

$637 \mathrm{NJ}$ ). Sections were then blocked overnight in normal donkey serum (Jackson ImmunoResearch;

638 West Grove, PA) diluted 1:20 in PBS containing 0.5\% bovine serum albumin, 0.1\% Triton-X

639100 , and $0.1 \%$ sodium azide, hereafter, PBTA at $4^{\circ} \mathrm{C}$. Next, primary antibodies anti-GFAP

640 (1:500; abcam; ab53554), anti-S100 (1:1000; DAKO; Z0311) were diluted in PBTA and

641 incubated overnight at $4^{\circ} \mathrm{C}$. Then, sections were rinsed $5 \times 5$ mins and $1 \times 1 \mathrm{hr}$ before

642 corresponding secondary antibodies along with the nuclear stain Hoechst33342 (1:5000;

643 Molecular Probes; $\mathrm{H}-3570$ ) were incubated overnight at 4ํㅡ. Lastly, secondary antibodies were

644 rinsed and sections mounted using Vectashield (Vector laboratories Inc; H-1200) and sealed

645 under \#0 coverslips.

High resolution wide-field mosaics of brain samples were then imaged with a 20x oil

647 immersion lens and an Olympus Fluoview 1000 laser scanning confocal microscope (Center

648 Valley, PA) at a pixel array of $800 \times 800$ and then registered using the bio-image software

649 Imago (Santa Barbara CA).

We then calculated glial cell density as a function of distance from the prism face. First, each mosaic was rotated so that the medial-lateral axis of the brain sample was aligned to be parallel with the horizontal axis of the image. Then each mosaic was cropped to remove

653 extraneous pixels outside of the imaged brain slice. Next, a line denoting the face of the prism

654 was manually drawn parallel to the dorsal-ventral axis aligned with the location of the prism

655 face. We then used a custom cell-counting algorithm that identified potential regions of interest

656 (ROls). We limited the ROls to be within the hippocampal formation in the brain slices. The

657 Euclidean distance between the closest point on the defined prism face and each ROl's centroid

658 was calculated. Afterward, a similar procedure was performed on the contralateral side of the

659 brain slice, with a mock "prism face" defined at symmetric coordinates to the true prism face, to

660 serve as a control. These steps were repeated for each channel of the mosaic.

661 After extracting each ROls distance from the prism face, we counted the number of cells

662 in each $50 \mu \mathrm{m}$ distance bin. To account for basal glial cell density, we calculated the percent

663 change of glial cell density on the prism side with respect to the control side. This procedure 
was repeated 1000 times using randomly sampled distances, with replacement, to bootstrap the

665 sample variance.

\section{Statistical Information}

667 Violin plots were made using an open source Matlab package ${ }^{95}$. Statistical tests for spine

668 morphological types were calculated using a one-way ANOVA. Reliability across laps was

669 tested with a two-sample Kolmogorov-Smirnov test. Comparisons between model fits for spatial

670 distribution of spatial information and place field width used a General Linear F-test.

\section{DATA AVAILABILITY}

Microperiscope designs can be found on our institutional lab website

(https://goard.mcdb.ucsb.edu/resources). Spine imaging data from Fig. 3 and neuronal response data from Figs. 5-6 are available on Dryad (DOI: TBA, final version will be uploaded upon manuscript acceptance). Code for spine analysis and place/speed cell identification is available on Github (DOI: TBA, final version will be uploaded upon manuscript acceptance).

\section{REFERENCES}

1. O'Keefe, J. \& Nadel, L. The Hippocampus as a Cognitive Map. (Oxford University Press, 1978).

2. Squire, L. R. Memory and the Hippocampuss: A Synthesis From Findings With Rats, Monkeys, and Humans. Psychol. Rev. 99, 195-231 (1992).

3. Tonegawa, S., Liu, X., Ramirez, S. \& Redondo, R. Memory Engram Cells Have Come of Age. Neuron vol. 87 918-931 (2015).

4. Ramon y Cajal, S. Histologie du système nerveux de l'homme et des vertèbres. (Maloine, 1911).

5. Gilbert, P. E., Kesner, R. P. \& Lee, I. Dissociating hippocampal subregions: A double dissociation between dentate gyrus and CA1. Hippocampus 11, 626-636 (2001).

6. Lee, I., Yoganarasimha, D., Rao, G. \& Knierim, J. J. Comparison of population coherence of place cells in hippocampal subfields CA1 and CA3. Nature 430, 456-459 (2004).

7. Leutgeb, S., Leutgeb, J. K., Treves, A., Moser, M. B. \& Moser, E. I. Distinct ensemble codes in hippocampal areas CA3 and CA1. Science 305, 1295-1298 (2004).

8. Leutgeb, J. K. et al. Progressive transformation of hippocampal neuronal representations in 'morphed' environments. Neuron 48, 345-358 (2005).

9. Leutgeb, J. K., Leutgeb, S., Moser, M. B. \& Moser, E. I. Pattern separation in the dentate gyrus and CA3 of the hippocampus. Science 315, 961-966 (2007). 
10. McHugh, T. J. et al. Dentate gyrus NMDA receptors mediate rapid pattern separation in the hippocampal network. Science 317, 94-99 (2007).

11. Nakashiba, T. et al. Young dentate granule cells mediate pattern separation, whereas old granule cells facilitate pattern completion. Cell 149, 188-201 (2012).

12. Kheirbek, M. A. et al. Differential control of learning and anxiety along the dorsoventral axis of the dentate gyrus. Neuron 77, 955-968 (2013).

13. Neunuebel, J. P. \& Knierim, J. J. CA3 retrieves coherent representations from degraded input: Direct evidence for CA3 pattern completion and dentate gyrus pattern separation. Neuron $\mathbf{8 1}$, 416-427 (2014).

14. Rennó-Costa, C., Lisman, J. E. \& Verschure, P. F. M. J. A Signature of Attractor Dynamics in the CA3 Region of the Hippocampus. PLoS Comput. Biol. 10, e1003641 (2014).

15. Frank, L. M., Stanley, G. B. \& Brown, E. N. Hippocampal plasticity across multiple days of exposure to novel environments. J. Neurosci. 24, 7681-7689 (2004).

16. Karlsson, M. P. \& Frank, L. M. Network dynamics underlying the formation of sparse, informative representations in the hippocampus. J. Neurosci. 28, 14271-14281 (2008).

17. Kemere, C., Carr, M. F., Karlsson, M. P. \& Frank, L. M. Rapid and continuous modulation of hippocampal network state during exploration of new places. PLoS One 8, e73114 (2013).

18. Larkin, M. C., Lykken, C., Tye, L. D., Wickelgren, J. G. \& Frank, L. M. Hippocampal output area CA1 broadcasts a generalized novelty signal during an object-place recognition task.

Hippocampus 24, 773-783 (2014).

19. Dong, C., Madar, A. D. \& Sheffield, M. E. J. Distinct place cell dynamics in CA1 and CA3 encode experience in new environments. Nat. Commun. 12, 1-13 (2021).

20. Hitti, F. L. \& Siegelbaum, S. A. The hippocampal CA2 region is essential for social memory. Nature 508, 88-92 (2014).

21. Okuyama, T., Kitamura, T., Roy, D. S., Itohara, S. \& Tonegawa, S. Ventral CA1 neurons store social memory. Science 353, 1536-1541 (2016).

22. Meira, T. et al. A hippocampal circuit linking dorsal CA2 to ventral CA1 critical for social memory dynamics. Nat. Commun. 9, 1-14 (2018).

23. Mankin, E. A. et al. Neuronal code for extended time in the hippocampus. Proc. Natl. Acad. Sci. U. S. A. 109, 19462-19467 (2012).

24. Hainmueller, T. \& Bartos, M. Parallel emergence of stable and dynamic memory engrams in the hippocampus. Nature 558, 292-296 (2018).

25. Dombeck, D. A., Harvey, C. D., Tian, L., Looger, L. L. \& Tank, D. W. Functional imaging of hippocampal place cells at cellular resolution during virtual navigation. Nat. Neurosci. 13, 1433- 
$1440(2010)$.

26. Ghosh, K. K. et al. Miniaturized integration of a fluorescence microscope. Nat. Methods 8, 871878 (2011).

27. Ziv, Y. et al. Long-term dynamics of CA1 hippocampal place codes. Nat. Neurosci. 16, 264-266 (2013).

28. Cai, D. J. et al. A shared neural ensemble links distinct contextual memories encoded close in time. Nature 534, 115-118 (2016).

29. Sheintuch, L. et al. Tracking the Same Neurons across Multiple Days in Ca2+ Imaging Data. Cell Rep. 21, 1102-1115 (2017).

30. Chia, T. H. \& Levene, M. J. Microprisms for in vivo multilayer cortical imaging. J. Neurophysiol. 102, 1310-1314 (2009).

31. Andermann, M. L. et al. Chronic Cellular Imaging of Entire Cortical Columns in Awake Mice Using Microprisms. Neuron 80, 900-913 (2013).

32. Low, R. J., Gu, Y. \& Tank, D. W. Cellular resolution optical access to brain regions in fissures: Imaging medial prefrontal cortex and grid cells in entorhinal cortex. Proc. Natl. Acad. Sci. U. S. A. 111, 18739-18744 (2014).

33. Gu, Y. et al. A Map-like Micro-Organization of Grid Cells in the Medial Entorhinal Cortex. Cell 175, 736-750.e30 (2018).

34. Kislin, M. et al. Flat-floored air-lifted platform: A new method for combining behavior with microscopy or electrophysiology on awake freely moving rodents. J. Vis. Exp. (2014) doi:10.3791/51869.

35. Go, M. A. et al. Place Cells in Head-Fixed Mice Navigating a Floating Real-World Environment. Front. Cell. Neurosci. 15, 618658 (2021).

36. Lee, H., Wang, C., Deshmukh, S. S. \& Knierim, J. J. Neural Population Evidence of Functional Heterogeneity along the CA3 Transverse Axis: Pattern Completion versus Pattern Separation. Neuron 87, 1093-1105 (2015).

37. Lu, L., Igarashi, K. M., Witter, M. P., Moser, E. I. \& Moser, M. B. Topography of Place Maps along the CA3-to-CA2 Axis of the Hippocampus. Neuron 87, 1078-1092 (2015).

38. Mankin, E. A., Diehl, G. W., Sparks, F. T., Leutgeb, S. \& Leutgeb, J. K. Hippocampal CA2 Activity Patterns Change over Time to a Larger Extent than between Spatial Contexts. Neuron 85, 190201 (2015).

39. Engert, F. \& Bonhoeffer, T. Dendritic spine changes associated with hippocampal long-term synaptic plasticity. Nature 399, 66-70 (1999).

40. Harris, K. M. Structure, development, and plasticity of dendritic spines. Curr. Opin. Neurobiol. 9, 
343-348 (1999).

41. Yuste, R. \& Bonhoeffer, T. Morphological changes in dendritic spines associated with long-term synaptic plasticity. Annual Review of Neuroscience vol. 24 1071-1089 (2001).

42. Yang, G., Pan, F. \& Gan, W. B. Stably maintained dendritic spines are associated with lifelong memories. Nature 462, 920-924 (2009).

43. Attardo, A., Fitzgerald, J. E. \& Schnitzer, M. J. Impermanence of dendritic spines in live adult CA1 hippocampus. Nature 523, 592-596 (2015).

44. Pfeiffer, T. et al. Chronic 2P-STED imaging reveals high turnover of dendritic spines in the hippocampus in vivo. Elife 7:e34700, (2018).

45. Feng, G. et al. Imaging neuronal subsets in transgenic mice expressing multiple spectral variants of GFP. Neuron 28, 41-51 (2000).

46. Chang, F. L. F. \& Greenough, W. T. Transient and enduring morphological correlates of synaptic activity and efficacy change in the rat hippocampal slice. Brain Res. 309, 35-46 (1984).

47. Rodriguez, A., Ehlenberger, D. B., Dickstein, D. L., Hof, P. R. \& Wearne, S. L. Automated threedimensional detection and shape classification of dendritic spines from fluorescence microscopy images. PLoS One 3, 1997 (2008).

48. Son, J., Song, S., Lee, S., Chang, S. \& Kim, M. Morphological change tracking of dendritic spines based on structural features. J. Microsc. 241, 261-272 (2011).

49. Lendvai, B., Stern, E. A., Chen, B. \& Svoboda, K. Experience-dependent plasticity of dendritic spines in the developing rat barrel cortex in vivo. Nature 404, 876-881 (2000).

50. Risher, W. C., Ustunkaya, T., Alvarado, J. S. \& Eroglu, C. Rapid golgi analysis method for efficient and unbiased classification of dendritic spines. PLoS One 9, e107591 (2014).

51. Fiala, J. C., Feinberg, M., Popov, V. \& Harris, K. M. Synaptogenesis via dendritic filopodia in developing hippocampal area CA1. J. Neurosci. 18, 8900-8911 (1998).

52. Stewart, M. G. et al. Chemically induced long-term potentiation increases the number of perforated and complex postsynaptic densities but does not alter dendritic spine volume in CA1 of adult mouse hippocampal slices. Eur. J. Neurosci. 21, 3368-3378 (2005).

53. Grutzendler, J., Kasthuri, N. \& Gan, W. B. Long-term dendritic spine stability in the adult cortex. Nature 420, 812-816 (2002).

54. Trachtenberg, J. T. et al. Long-term in vivo imaging of experience-dependent synaptic plasticity in adult cortex. Nature 420, 788-794 (2002).

55. Holtmaat, A. J. G. D. et al. Transient and persistent dendritic spines in the neocortex in vivo. Neuron 45, 279-291 (2005).

56. van Dijk, M. T. \& Fenton, A. A. On How the Dentate Gyrus Contributes to Memory Discrimination. 
Neuron 98, 832-845.e5 (2018).

57. Stefanini, F. et al. A Distributed Neural Code in the Dentate Gyrus and in CA1. Neuron 107, 703716.e4 (2020).

58. Chen, T. W. et al. Ultrasensitive fluorescent proteins for imaging neuronal activity. Nature 499, 295-300 (2013).

59. Daigle, T. L. et al. A Suite of Transgenic Driver and Reporter Mouse Lines with Enhanced BrainCell-Type Targeting and Functionality. Cell 174, 465-480.e22 (2018).

60. Aronov, D. \& Tank, D. W. Engagement of Neural Circuits Underlying 2D Spatial Navigation in a Rodent Virtual Reality System. Neuron 84, 442-456 (2014).

61. Aghajan, Z. M. et al. Impaired spatial selectivity and intact phase precession in two-dimensional virtual reality. Nat. Neurosci. 18, 121-128 (2015).

62. Kropff, E., Carmichael, J. E., Moser, M. B. \& Moser, E. I. Speed cells in the medial entorhinal cortex. Nature 523, 419-424 (2015).

63. Iwase, M., Kitanishi, T. \& Mizuseki, K. Cell type, sub-region, and layer-specific speed representation in the hippocampal-entorhinal circuit. Sci. Rep. 10, 1-23 (2020).

64. Góis, Z. H. T. D. \& Tort, A. B. L. Characterizing Speed Cells in the Rat Hippocampus. Cell Rep. 25, 1872-1884.e4 (2018).

65. Claiborne, B. J., Amaral, D. G. \& Cowan, W. M. A light and electron microscopic analysis of the mossy fibers of the rat dentate gyrus. J. Comp. Neurol. 246, 435-458 (1986).

66. Ishizuka, N., Weber, J. \& Amaral, D. G. Organization of intrahippocampal projections originating from CA3 pyramidal cells in the rat. J. Comp. Neurol. 295, 580-623 (1990).

67. Li, X. - G, Somogyi, P., Ylinen, A. \& Buzsáki, G. The hippocampal CA3 network: An in vivo intracellular labeling study. J. Comp. Neurol. 339, 181-208 (1994).

68. Ishizuka, N., Cowan, W. M. \& Amaral, D. G. A quantitative analysis of the dendritic organization of pyramidal cells in the rat hippocampus. J. Comp. Neurol. 362, 17-45 (1995).

69. Witter, M. P. Intrinsic and extrinsic wiring of CA3: Indications for connectional heterogeneity. Learning and Memory vol. 14 705-713 (2007).

70. Levene, M. J., Dombeck, D. A., Kasischke, K. A., Molloy, R. P. \& Webb, W. W. In Vivo Multiphoton Microscopy of Deep Brain Tissue. J. Neurophysiol. 91, 1908-1912 (2004).

71. Barretto, R. P. J. et al. Time-lapse imaging of disease progression in deep brain areas using fluorescence microendoscopy. Nat. Med. 17, 223-229 (2011).

72. Chambers, A. R. \& Rumpel, S. A stable brain from unstable components: Emerging concepts and implications for neural computation. Neuroscience vol. 357 172-184 (2017). 
73. Mongillo, G., Rumpel, S. \& Loewenstein, Y. Intrinsic volatility of synaptic connections - a challenge to the synaptic trace theory of memory. Current Opinion in Neurobiology vol. 46 7-13 (2017).

74. Kentros, C. G., Agnihotri, N. T., Streater, S., Hawkins, R. D. \& Kandel, E. R. Increased attention to spatial context increases both place field stability and spatial memory. Neuron 42, 283-295 (2004).

75. Kinsky, N. R., Sullivan, D. W., Mau, W., Hasselmo, M. E. \& Eichenbaum, H. B. Hippocampal Place Fields Maintain a Coherent and Flexible Map across Long Timescales. Curr. Biol. 28, 35783588.e6 (2018).

76. Mau, W., Hasselmo, M. E. \& Cai, D. J. The brain in motion: How ensemble fluidity drives memoryupdating and flexibility. Elife 9:e63550, 1-24 (2020).

77. Wilson, M. A. \& McNaughton, B. L. Dynamics of the hippocampal ensemble code for space. Science 261, 1055-1058 (1993).

78. Danielson, N. B. et al. In Vivo Imaging of Dentate Gyrus Mossy Cells in Behaving Mice. Neuron 93, 552-559.e4 (2017).

79. GoodSmith, D. et al. Spatial Representations of Granule Cells and Mossy Cells of the Dentate Gyrus. Neuron 93, 677-690.e5 (2017).

80. Senzai, Y. \& Buzsáki, G. Physiological Properties and Behavioral Correlates of Hippocampal Granule Cells and Mossy Cells. Neuron 93, 691-704.e5 (2017).

81. Mongillo, G., Rumpel, S. \& Loewenstein, Y. Inhibitory connectivity defines the realm of excitatory plasticity. Nat. Neurosci. 21, 1463-1470 (2018).

82. Sheffield, M. E. J. \& Dombeck, D. A. Calcium transient prevalence across the dendritic arbour predicts place field properties. Nature 517, 200-204 (2015).

83. Sheffield, M. E. J., Adoff, M. D. \& Dombeck, D. A. Increased Prevalence of Calcium Transients across the Dendritic Arbor during Place Field Formation. Neuron 96, 490-504.e5 (2017).

84. Adoff, M. D. et al. The functional organization of excitatory synaptic input to place cells. Nat. Commun. 12, (2021).

85. Jia, H., Rochefort, N. L., Chen, X. \& Konnerth, A. Dendritic organization of sensory input to cortical neurons in vivo. Nature 464, 1307-1312 (2010).

86. Wilson, D. E., Whitney, D. E., Scholl, B. \& Fitzpatrick, D. Orientation selectivity and the functional clustering of synaptic inputs in primary visual cortex. Nat. Neurosci. 19, 1003-1009 (2016).

87. Wang, Q. et al. The Allen Mouse Brain Common Coordinate Framework: A 3D Reference Atlas. Cell 181, 936-953.e20 (2020).

88. Paxinos, G. \& Franklin, K. B. J. The Mouse Brain in Stereotaxic Coordinates. (Academic Press, 
2001).

89. Zipfel, W. R., Williams, R. M. \& Webb, W. W. Nonlinear magic: Multiphoton microscopy in the biosciences. Nature Biotechnology vol. 21 1369-1377 (2003).

90. Berens, P. CircStat: A MATLAB Toolbox for Circular Statistics. J. Stat. Softw. 31, 1-21 (2009).

91. Pachitariu, M. et al. Suite2p: beyond 10,000 neurons with standard two-photon microscopy. bioRxiv 061507 (2016) doi:10.1101/061507.

92. Friedrich, J., Zhou, P. \& Paninski, L. Fast online deconvolution of calcium imaging data. PLoS Comput. Biol. 13, (2017).

93. Holtmaat, A. \& Svoboda, K. Experience-dependent structural synaptic plasticity in the mammalian brain. Nature Reviews Neuroscience vol. 10 647-658 (2009).

94. Skaggs, W., McNaughton, B. \& Gothard, K. An Information-Theoretic Approach to Deciphering the Hippocampal Code. Adv. Neural Inf. Process. Syst. 5, 1030--1037 (1993).

95. Bechtold, B. Violin Plots for Matlab, Github Project. (2016).

\section{ACKNOWLEDGEMENTS}

We would like to thank Caleb Kamere and Eliott Levy for comments on the manuscript. This work was supported by NSF (M.J.G., S.L.S., NeuroNex \#1707287), NIH (M.J.G., S.L.S., R01NS121919), the Whitehall Foundation (M.J.G.), and the Larry Hillblom foundation (M.J.G.).

\section{AUTHOR CONTRIBUTIONS}

W.T.R. and M.J.G. designed the experiments; M.J.G. performed the surgical implants; W.T.R., N.S.W., L.M., and M.J.G. conducted the imaging experiments and analyzed the data; G.L. and T.D.M. performed immunohistochemistry and K.K.S. analyzed the resulting images; C.H.Y. and S.L.C. performed optical modeling; W.T.R., N.S.W., and M.J.G. wrote the manuscript.

\section{COMPETING INTERESTS}

The authors declare no competing financial interests.

\section{MATERIALS AND CORRESPONDENCE}

Correspondence and material requests should be directed to michael.goard@lifesci.ucsb.edu. 